\title{
The Geometry of Real Sine-Gordon Wavetrains
}

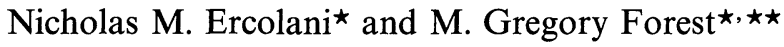 \\ Department of Mathematics, The Ohio State University, Columbus, OH 43210, USA
}

\begin{abstract}
The characterization of real, $N$ phase, quasiperiodic solutions of the sine-Gordon equation has been an open problem. In this paper we achieve this result, employing techniques of classical algebraic geometry which have not previously been exploited in the soliton literature. A significant by-product of this approach is a natural algebraic representation of the full complex isospectral manifolds, and an understanding of how the real isospectral manifolds are embedded. By placing the problem in this general context, these methods apply directly to all soliton equations whose multiphase solutions are related to hyperelliptic functions.
\end{abstract}

\section{Introduction}

The $N$ phase quasiperiodic solutions of the Korteweg-de Vries (KdV), sineGordon (sG), and sinh-Gordon (sh-G) equations have explicit representations in terms of theta functions of $N$ variables [7,8]. The exact integration for this class of solutions is achieved through the spectral theory of an associated linear system: the nonlinear evolution equation generates one of an infinite family of involutive isospectral flows for the $x$-eigenvalue problem. An important distinction, however, is that the spectral problem for real $N$ phase potentials is selfadjoint for $\mathrm{KdV}$ and sh-G, but non-selfadjoint for sine-Gordon. The methods in [14] for KdV and in [5] for sh-G are not amenable to non-selfadjoint problems, so the real $N$ phase sine-Gordon theory remained open.

The point of view taken here is that the formal inverse spectral structure of all these partial differential equations (p.d.e.'s) is the same at the level of complex quasiperiodic potentials. We deduce the structure of the real isospectral class as a subset of the full complex isospectral class. This common structure of $N$ phase potentials consists of: $N$ dynamical variables, $\boldsymbol{\mu}=\left(\mu_{1}, \ldots, \mu_{N}\right)$, which are Dirichlet

* Supported in part by NSF Grant No. MCS-8202288

$\star \star$ Supported in part by NSF Grant No. MCS-8002969 
eigenvalues for strictly periodic potentials, and the isospectral data $\left\{E_{j}\right\}_{j=1}^{2 N}$, which consists of simple periodic eigenvalues for periodic potentials.

The solution of the p.d.e. is determined from this spectral data; for example,

$$
\begin{aligned}
u_{\mathrm{KdV}} & =\sum_{j} E_{j}-2 \sum_{j} \mu_{j}, \\
u_{\mathrm{sG}} & =i \ln \left(\prod_{j} \mu_{j} / \sqrt{\prod_{j} E_{j}}\right) .
\end{aligned}
$$

Moreover, the flows of $\boldsymbol{\mu}(x, t)$ have provided natural dynamical coordinates for the real isospectral classes of $\mathrm{KdV}$ and sh-G; classical selfadjoint oscillation theorems constrain the real flows of $\mu_{j}$ to oscillate in fixed real bands between $E_{2 j-1}$ and $E_{2 j}$. In contrast, while the reality constraints on $\left\{E_{j}\right\}$ for sine-Gordon are simple (Sect. II.B), the $\boldsymbol{\mu}$ trajectories are quite complicated and, in fact, do not lie on fixed curves (for $N>1$ ) in the complex plane.

It is natural (Sects. I.D and E) to regard each $\mu_{j}$ as a coordinate on a hyperelliptic Riemann surface, $\mathscr{R}$, of genus $N$, with branch points at the invariant $\left\{E_{j}\right\}$. (We assume $\mathscr{R}$ is nonsingular; that is, we exclude higher dimensional "separatrices.")

By studying the geometry of the $\boldsymbol{\mu}$ variables in this setting, we determine the number of connected components of the real isospectral manifold associated to each $\left\{E_{j}\right\}_{j=1}^{2 N}$, we show each component is a real $N$ dimensional subtorus [Eq.(II.9)] of the Jacobian of $\mathscr{R}, \mathscr{J}(\mathscr{R})$, and we describe the physical characteristics of the waves in a given component (Propositions II.2 and II.3). Furthermore, we show the $\boldsymbol{\mu}$ variables coordinatize the real $N$ phase isospectral manifolds, and we deduce the $\boldsymbol{\mu}$ trajectories under real (sG) flows. For the $N=2$ case, we go on to derive explicit expressions for $\left(\mu_{1}, \mu_{2}\right)$ and then we provide computer plots of these exact $\boldsymbol{\mu}$ flows.

The algebro-geometric setting for our analysis consists of three stages:

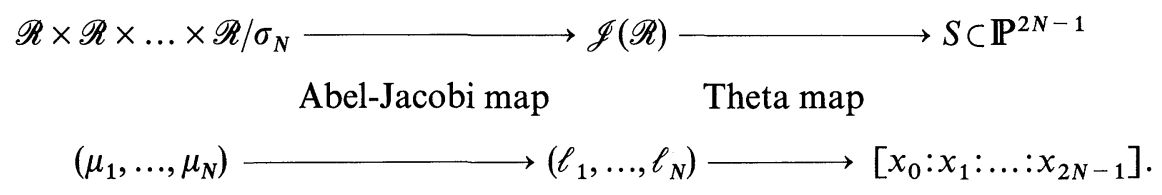

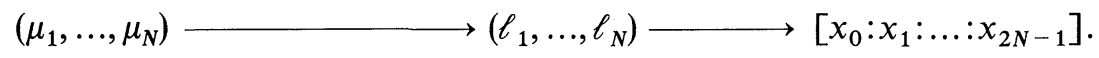

The first level is $N$ unordered copies of $\mathscr{R}$. The second level, the Jacobian, $\mathscr{J}(\mathscr{R})$, is where the complex phases $\ell(x, t)$ (linear in $x$ and $t$ ) are defined. These two levels are connected by the Abel-Jacobi map. This much is well-documented in the literature. Our contribution is the third level, which is the Kummer variety $S$.

The maps in the above diagram provide a means of translating expressions involving theta functions on $\mathscr{J}$ into rational functions on $S$. As mentioned earlier, in the $N=2$ case, we explicitly carry out this translation. The result is the inversion formulae for $\left(\mu_{1}, \mu_{2}\right)$, which are algebraic (linear fractional) functions of the coordinates $x_{0}, x_{1}, x_{2}, x_{3}$ on $S$ :

$$
\mu_{1} \mu_{2}=\sqrt{E_{1} E_{2} E_{3} E_{4}} \frac{\delta x_{0}+\gamma x_{1}-\beta x_{2}-\alpha x_{3}}{\delta x_{0}-\gamma x_{1}+\beta x_{2}-\alpha x_{3}},
$$

and a similar formula (III.13) for $\mu_{1}+\mu_{2} . \alpha, \beta, \gamma, \delta$ are constants which depend only on the $E_{i}$. The $x_{i}$, coordinates on $S$, are quadratic theta functions. 
These $N=2$ (and analogous $N>2$ ) formulae are valid globally - for any $\mathscr{R}$ and for all complex flows on $\mathscr{J}(\mathscr{R})$. We then show how the reality constraints on $\left\{E_{j}\right\}$, and thus on $\mathscr{R}$, translate into constraints at all three levels of our diagram.

We do not assume the reader is an expert on the geometry used here; our presentation is self-contained and well referenced.

\section{Preliminaries}

We begin by summarizing several well-known developments in the study of $N$ phase, quasiperiodic solutions of the sine-Gordon (sG) equation.

\section{A. Exact Integration}

The exact integration of the (sG) equation,

$$
u_{t t}-u_{x x}+\sin u=0, \quad-\infty<x<\infty,
$$

is achieved through inverse spectral theory (IST) of a linear eigenvalue problem in $x$, together with a linear system for the time flow of the eigenfunction. Equation (I.1) arises as the compatibility condition between these systems. This method for (sG) is due to Lamb [1] and was clarified independently by Ablowitz, Kaup, Newell, and Segur [2] (AKNS) and Faddeev and Takhtajian [3] (FT). We use the (FT) linear system; for eigenfunction $\psi=\left(\psi_{1}, \psi_{2}\right)^{T}$,

$$
A \underset{t}{x}+\frac{i}{4} w B \Psi \pm \frac{1}{16 \sqrt{E}} C \psi=\sqrt{E} \boldsymbol{\psi}
$$

where

$$
A=\left(\begin{array}{rr}
0 & -1 \\
1 & 0
\end{array}\right), \quad B=\left(\begin{array}{ll}
0 & 1 \\
1 & 0
\end{array}\right), \quad C=\left(\begin{array}{cc}
\exp (i u) & 0 \\
0 & \exp (-i u)
\end{array}\right) .
$$

Remark 1. Observe that the spectral parameter $\sqrt{E}$ is multivalued. This occurs because (I.2) is the reduction of a fourth order system $[4,5]$ which expresses (sG) in Lax form, $\dot{Q}=[\kappa, Q]$.

One easily checks the compatibility of (I.1), (I.2):

$$
\boldsymbol{\psi}_{x t}=\boldsymbol{\psi}_{t x} \quad \text { iff } u, w \text { satisfy }\left\{\begin{array}{l}
u_{x}+u_{t}=w \\
w_{x}-w_{t}=\sin u
\end{array}\right.
$$

The boundary conditions in (I.2a) are shared with the potentials $u, w$. In this paper we are interested in periodic and quasiperiodic potentials with finite $(N)$ degrees of freedom. These are referred to in the IST literature as $N$ phase quasiperiodic potentials, or as $N$ phase wavetrains in the context of solutions of a pde.

Definition 1. An $N$ phase quasiperiodic potential $u(x, t)$ satisfies:

(i) The entire $x, t$ dependence can be expressed in terms of $N$ phases, $\theta_{i}(x, t)$, linear in $x$ and $t$,

$$
\theta_{i}(x, t)=\kappa_{i} x+\omega_{i} t, \quad i=1, \ldots, N ;
$$


(ii) $u(x, t)=u(\theta(x, t))$ is periodic $(\bmod 2 \pi)$ of finite period in each phase,

$$
u\left(\theta_{1}, \ldots, \theta_{j}+2 \pi, \ldots, \theta_{N}\right)=u\left(\theta_{1}, \ldots, \theta_{j}, \ldots, \theta_{N}\right) \bmod 2 \pi .
$$

If the phases $\theta_{i}$ are real, $\kappa$ are the spatial wavenumbers and $\omega$ are the temporal frequencies.

Definition 2. Let $u(x, t)=u(\theta(x, t))$ be an $N$ phase quasiperiodic potential, with prescribed $x$-period $L$. Then $u$ is an $N$ phase periodic potential iff $\kappa_{1}, \ldots, \kappa_{N}$ are each rational multiples of $2 \pi / L$.

Remark 2. In the periodic case, condition (ii) of Definition 1 excludes separatrices, which do in fact arise in the phase space of all periodic potentials of (I.2a). (In contrast, this does not occur for periodic Hill potentials.) Already in the $N=1$ case of traveling waves, the phase space of $(\mathrm{sG})$ is the same as the pendulum, $\left(\omega^{2}-\kappa^{2}\right) u_{\theta \theta}$ $+\sin u=0$. The two branches of the separatrix $\Gamma$ correspond to the infinite period kink and antikink solitons of (sG) [4] (see Sect. I.G).

Fig. 1. $N=1$ phase portrait

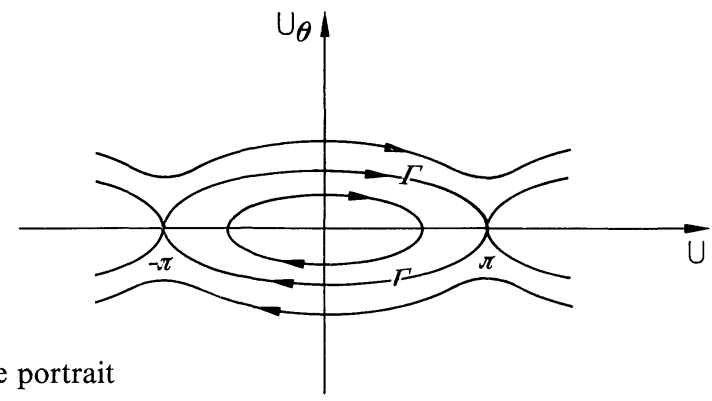

For periodic potentials, as $u, w$ evolve in $t$ according to the (sG) equation, the simple periodic spectrum, $\Sigma^{(s)}$, of (I.2a) remains invariant: (sG) generates an isospectral flow for (I.2a). (sG) is the first of an infinite hierarchy of commuting flows which preserves $\Sigma^{(s)}$. A discussion of the connections between the spectrum of (I.2a) and the corresponding solutions of (sG) can be found in [4]. One pertinent fact is:

For real potentials $u, w$, the simple periodic spectrum $\Sigma^{(s)}$ consists of distinct pairs $E_{2 j}, E_{2 j-1}$ which are either real and negative, $E_{2 j}<E_{2 j-1}<0$, or occur in complex conjugate pairs, $E_{2 j}^{*}=E_{2 j-1}$.

\section{B. Squared Eigenfunctions}

The construction of $N$ phase solutions is remarkably streamlined if one replaces (I.2) by an equivalent system for quadratic or squared eigenfunctions. Let $\boldsymbol{\psi}=\left(\psi_{1}, \psi_{2}\right)^{T}, \boldsymbol{\varphi}=\left(\varphi_{1}, \varphi_{2}\right)^{T}$ denote two independent solutions of (I.2); then define

$$
f=\frac{-i}{2}\left(\psi_{1} \varphi_{2}+\psi_{2} \varphi_{1}\right), \quad g=\psi_{1} \varphi_{1}, \quad h=-\psi_{2} \varphi_{2}
$$


It follows by direct computation that (I.2) is equivalent to the squared eigenfunction linear system:

$$
\begin{aligned}
& f_{x}=i\left(\sqrt{E} \mp \frac{e^{i u}}{16 \sqrt{E}}\right) g+i\left(\sqrt{E} \mp \frac{e^{-i u}}{16 \sqrt{E}}\right) h, \\
& g_{x}=\frac{-i}{2} w g+2 i\left(\sqrt{E} \mp \frac{e^{-i u}}{16 \sqrt{E}}\right) f, \\
& h_{x}=\frac{i}{2} w h+2 i\left(\sqrt{E} \mp \frac{e^{i u}}{16 \sqrt{E}}\right) f .
\end{aligned}
$$

Many fundamental results about the $(\mathrm{sG})$ equation at the exact and perturbative level are easily deduced from this system $[4,6,17,18]$, as indicated below.

C. The Finite-Band Inverse Spectral Problem

With the simple periodic spectrum $\Sigma^{(s)}$ of (I.2a) given by

$$
\Sigma_{N}^{(s)}=\left\{E_{1}, \ldots, E_{2 N}, \text { all distinct }\right\},
$$

(i) determine the full class of potentials $u, w$ with this prescribed $\Sigma^{(s)}$ (the isospectral class);

(ii) characterize the real isospectral class of $\Sigma^{(s)}$ constrained by (I.3).

Partial results on this problem for (sG) may be found in [4-9]; $u \equiv u_{N}(x, t)$ is shown to be an $N$ phase quasiperiodic potential admitting several equivalent representations, summarized next.

Remark $2^{\prime} . E_{j}$ are distinct, by assumption. Separatrices in the periodic phase space can occur when a pair of $E_{j}$ collides.

D. Polynomial Squared Eigenfunctions

and the $\boldsymbol{\mu}$-Representation of $u_{N}(x, t)$

A very concise method, due to Date [10], for the construction of $u_{N}$ shows that for $\Sigma^{(s)}$ given by (I.5) the squared eigenfunctions $f, g, h$ are, up to normalization, polynomial in $\sqrt{E}$,

$$
f=\frac{1}{\sqrt{E}} \sum_{j=1}^{N} f_{j}(x, t) E^{j}, \quad g=\sum_{j=0}^{N} g_{j}(x, t) E^{j}, \quad h=\sum_{j=0}^{N} h_{j}(x, t) E^{j},
$$

if and only if $u(x, t)$ is an $N$ phase wavetrain, with

$$
e^{-i u(x, t)}=\frac{\prod_{j=1}^{N} \mu_{j}(x, t)}{\sqrt{\prod_{1}^{2 N} E_{j}}} .
$$

The variables $\mu_{j}(x, t), j=1, \ldots, N$, are the $N$ zeroes of the squared eigenfunction $g$,

$$
g(x, t)=\prod_{j: 1}^{N}\left(E-\mu_{j}(x, t)\right),
$$


and they parametrize the $N$ degrees of freedom in the sine-Gordon solution $u_{N}$. These variables $\boldsymbol{\mu}$, for periodic potentials, are exactly the auxiliary spectrum discussed by McKean [5].

The dynamical behaviour of the $\boldsymbol{\mu}$-variables follows from (I.4) (see [4]):

$$
\left(\mu_{j}\right)_{t}=2 i\left(1 \mp \frac{(-1)^{N} \prod_{\ell \neq j} \mu_{\ell}}{16 \sqrt{\prod_{k=1}^{2 N} E_{k}}}\right) \frac{R\left(\mu_{j}\right)}{\prod_{\ell \neq j}\left(\mu_{j}-\mu_{\ell}\right)},
$$

where

$$
R^{2}(\mu)=\prod_{k=0}^{2 N}\left(\mu-E_{k}\right), \quad E_{0}=0 .
$$

The relations (I.7), (I.9) are referred to as the $\mu$-representation of $u_{N}(x, t)$. Of course, this is an implicit representation; to obtain explicit, closed-form solutions, the $\boldsymbol{\mu}$-equations (I.9) must be integrated.

\section{E. Riemann Surfaces and the N-Phase Representation of $u_{N}(x, t)$}

From the representation above, it is apparent that each $\mu_{j}(x, t)$ resides naturally on the genus $N$, hyperelliptic Riemann surface $\mathscr{R}$ defined by (I.9b). In fact, the system (I.9) of ordinary differential equations can be linearized by the Abel-Jacobi map in the following way.

Choose a canonical set of cycles, $a_{j}, b_{j}, j=1, \ldots, N$ on $\mathscr{R}$. (Figure 2 depicts these cycles for an example.) Then introduce a normalized basis, $\left\{\psi_{j}, j=1, \ldots, N\right\}$, of holomorphic differentials on $\mathscr{R}$,

$$
\psi_{j}=\sum_{m=1}^{N} c_{j m} E^{N-m} \frac{d E}{R(E)},
$$

where the coefficients $c_{j m}$ are uniquely determined by the normalization

$$
\oint_{a_{k}} \psi_{j}=\delta_{k j}
$$

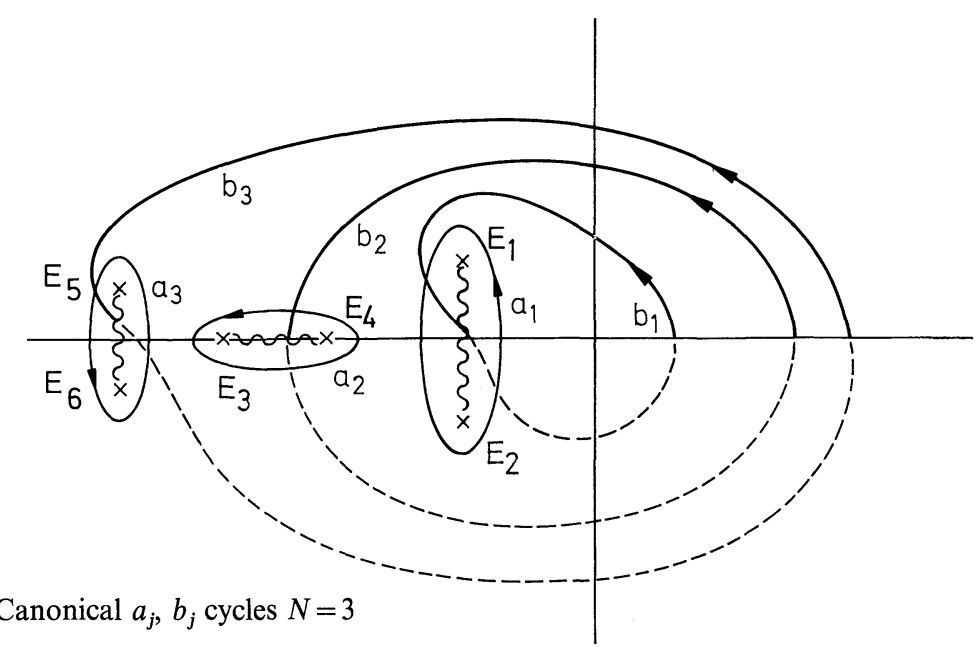

Fig. 2. Canonical $a_{j}, b_{j}$ cycles $N=3$ 
Denote the columns of $a_{j}$ periods of $\psi=\left(\psi_{1}, \ldots, \psi_{N}\right)^{T}$ by

$$
\oint_{a_{j}} \psi=e_{j} .
$$

The period matrix $B=\left(B_{i j}\right)$ is defined by

$$
B_{i j}=\oint_{b_{i}} \psi_{j}
$$

Denote the columns of $B$ by

$$
B_{j}=\oint_{b_{j}} \psi
$$

Next, change variables from $\boldsymbol{\mu}$ to $\ell$ via the Abel-Jacobi map:

$$
\ell(\boldsymbol{\mu})=\sum_{k=1}^{N} \int_{\dot{\mu}_{k}}^{\mu_{k}} \boldsymbol{\psi}
$$

where $\left(\dot{\mu}_{1}, \ldots, \dot{\mu}_{N}\right)$ represents a fixed $N$-tuple of points on $\mathscr{R}$. Geometrically, this situation is depicted by

$$
\begin{aligned}
& \Psi: \quad \begin{array}{ccc}
\boldsymbol{\mu} & \text { Abel-Jacobi } \\
& & \text { transform } \\
& &
\end{array} \\
& (\mathscr{R} \times \mathscr{R} \times \ldots \times \mathscr{R}) / \sigma_{N} \quad \mathscr{J}(\mathscr{R})=\mathbb{C}^{N} / \Lambda, \\
& \Lambda=\left\{e_{1}, \ldots, e_{N}, B_{1}, \ldots, B_{N}\right\} .
\end{aligned}
$$

$\Psi$ is the Abel-Jacobi transform (Sect. III.C), which is a map from the $\boldsymbol{\mu}$ space ( $N$ unordered copies of $\mathscr{R})$ to the space $\mathscr{J}(\mathscr{R})$ of complex phases $\ell$, the Jacobian of $\mathscr{R}$. It is clear from (I.11a) that $\ell$ is only well-defined modulo the periods of $\psi$, which form the lattice $\Lambda$. ( $\sigma_{N}$ is the group of permutations on $N$ symbols.)

It follows from (I.9) that each $\ell_{j}(\boldsymbol{\mu})$ is linear in $x$ and $t$ [4] (they are phases); explicitly,

$$
\ell_{j}(\boldsymbol{\mu})=-2 i\left[\left(c_{j 1}-\frac{(-1)^{N}}{16 \sqrt{\pi E_{k}}} c_{j N}\right) x+\left(c_{j 1}+\frac{(-1)^{N}}{16 \sqrt{\pi E_{k}}} c_{j N}\right) t\right]+\ell_{j}^{0} .
$$

Thus, the $x, t$ flows of the $\boldsymbol{\mu}$ variables linearize on the Jacobian of $\mathscr{R}$ in terms of $N$ complex phases $\ell(x, t)$. By virtue of the $\boldsymbol{\mu}$ representation, (I.7), the inverse problem for $\Sigma_{N}^{(s)}$ in (I.5) is solved at the level of complex potentials. The isospectral class is an $N$-dimensional complex torus, consisting of $N$-phase potentials. This torus is parametrized by $\ell$ or $\boldsymbol{\mu}$. (See Birnir [15] for the complex KdV case.)

The remaining questions are:

How are the flows of real potentials embedded in $\mathscr{J}(\mathscr{R})$ ?

What is the behavior of the auxiliary spectra $\mu$ restricted to these real flows; do they coordinatize the real isospectral class?

\section{F. Jacobi Inversion and the Theta-Function Representation}

To determine $\boldsymbol{\mu}$ explicitly as a function of the phases $\ell(x, t)$, one must invert (I.11). This is the classical Jacobi inversion problem. The solution involves the Riemann 
theta function, $\Theta(\mathbf{z} ; B) \equiv \Theta(\mathbf{z})$. This is an automorphic function of $N$ complex variables $\mathbf{z} \in \mathscr{J}(\mathscr{R})$ constructed from the period matrix $B$ of $\mathscr{R}$,

$$
\Theta(\mathbf{z})=\sum_{\boldsymbol{k} \in \mathbb{Z}^{N}} \exp \{i \pi(\langle B \mathbf{k}, \mathbf{k}\rangle+2\langle\mathbf{z}, \mathbf{k}\rangle)\} .
$$

The representation of meromorphic functions on $\mathscr{J}(\mathscr{R})$ by theta quotients [12], combined with (I.7), yields the theta-function representation of the $N$ phase wavetrain:

$$
\begin{gathered}
u_{N}(x, t)=2 i \ln \left[\frac{\Theta\left(\ell+\Delta+\frac{1}{2}\right)}{\Theta(\ell+\Delta)}\right], \\
\Delta=\text { Riemann's constant }, \\
\frac{1}{2} \equiv\left(\frac{1}{2}, \frac{1}{2}, \ldots, \frac{1}{2}\right) .
\end{gathered}
$$

The reality questions posed above in (I.E) now translate into a function theory problem.

Characterize all $\ell(x, t)$ of the form (I.11b) such that

$$
\left|\Theta\left(\ell+\Delta+\frac{1}{2}\right)\right|=|\Theta(\ell+\Delta)| .
$$

\section{G. Motivation: $N=1$, the Pendulum}

We will state the answers to the reality questions raised above in the simplest case, $N=1$. These results are taken from [4]. For real $u, w$ there are two choices of $\Sigma_{N=1}^{(s)}$ :

Case 1. $\Sigma^{(s)}=\left\{E_{1}, E_{2}=E_{1}^{*}\right)$.

Case 2. $\Sigma^{(s)}=\left\{E_{2}<E_{1}<0\right\}$.

Case 1 corresponds to purely oscillatory solutions (low energy) with phase curves interior to the separatrix. Case 2 corresponds to periodic $\bmod 2 \pi$ solutions, and there are two disjoint phase curves for each choice of $\Sigma^{(s)}$. This is familiar from the pendulum, which for large energy winds around and around either way. In other words, the real $N=1$ isospectral class for Case 1 is one circle, for Case 2 is two disjoint circles.

We now translate these results into our geometric context, with all terms as defined above.

Case 1. $\operatorname{Re}\left(\ell_{0}\right)=0, \ell(x, t) \in i \mathbb{R},|\mu|=\left|E_{1}\right|, \mu$ cycle $= \pm(a-2 b)$.

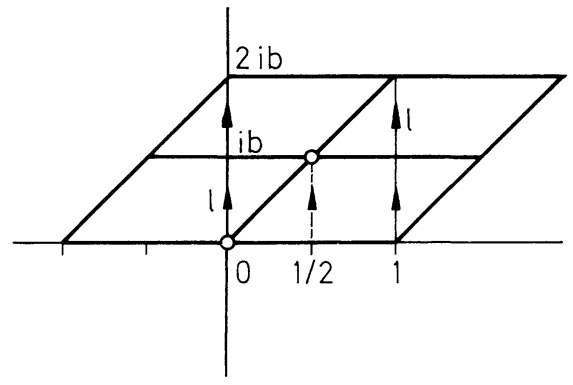

Fig. 3a. $\quad \ell \in \mathscr{J}(\mathscr{R})=\mathbb{C} /\left\{1, \frac{1}{2}+\mathrm{ib}\right\}$ $\{\ell(x, t) \mid x, t \in \mathbb{R}\} \equiv\left\{\ell(x, t)+\frac{1}{2} \mid x, t \in \mathbb{R}\right\}$

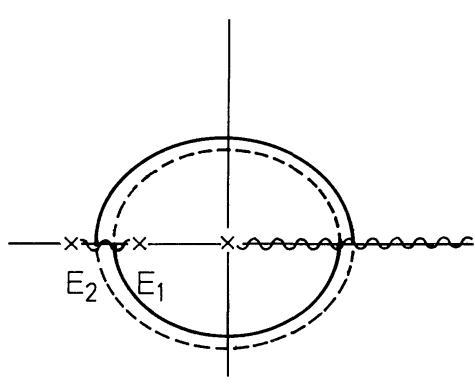

$\mu$-cycle on $\mathscr{R}$ 
Note there is one connected component in $\mathscr{J}(\mathscr{R})$ and one connected cycle of $\mu \in \mathscr{R}$ [the cycles $+(a-2 b),-(a-2 b)$ intersect at $\left.E_{1}, E_{2}\right]$.

Case 2. $\operatorname{Re}\left(\ell_{0}\right)= \pm \frac{1}{4}, \ell(x, t)-\ell_{0} \in i \mathbb{R},|\mu|=\sqrt{E_{1} E_{2}}, \mu$ cycle $\sim \pm b$.

Fig. 3b. $\quad \ell \in \mathscr{J}(\mathscr{R})=\mathbb{C} /\{1, \mathrm{ib}\}$

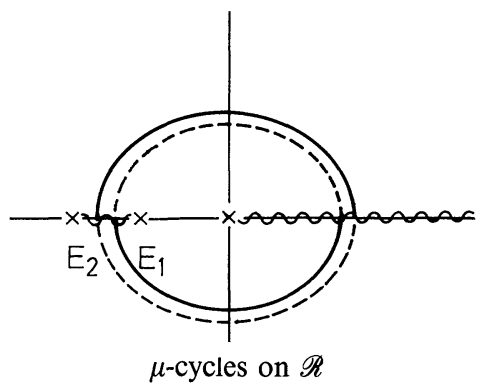

Note there are two connected components in $\mathscr{J}(\mathscr{R})$ and two disjoint $\mu$ cycles on $\mathscr{R}$, $\pm b$, one on each sheet.

Remark. For future reference (Remark 3 at the end of Sect. II), note that each $\ell_{0}$ in Cases 1,2 also yields a real connected component if we replace $\ell_{0}$ by $\ell_{0}+\frac{1}{2}$. In Case 2, it is clear from the figure that the two disjoint flows on $\mathscr{J}(\mathscr{R})$ are interchanged under translation by $\frac{1}{2}$. In Case 1 , notice that the real flow of $\ell_{0}=\frac{1}{2}$ on $\mathscr{J}$ hits $\frac{1}{2}+i b$, which equals 0 on $\mathscr{J}$, thus these are identical on $\mathscr{J}$.

From the $\Theta$ representation (I.13), it is obvious that $\ell_{0} \rightarrow \ell_{0}+\frac{1}{2}$ corresponds to $u \rightarrow-u$. In the phase space for the pendulum, Fig. 1, these geometric facts are merely reproducing the fact that for low energies (Case 1) the flows are invariant to $u \rightarrow-u$, but for large energy (Case 2) the kink and antikink modes are disjoint flows but related by $u \rightarrow-u$.

\section{Real $\mathbf{N}$-Phase sine-Gordon Wavetrains}

We begin with some historical background. Forest and McLaughlin [4] pointed out the reality problems for $N$-phase (sG) wavetrains, and solved the $N=1$ case completely, indicating the multiple components alluded to above. McKean [5] proved that for the $(\mathrm{sG})$ Hamiltonian sufficiently small, the real isospectral class is one real $N$ torus. (As we will show, this is included in the case of $\Sigma^{(s)}$ containing $N$ conjugate pairs.) Forest and McLaughlin [6] illustrated for $N=2$ how to use Riemann surface symmetries to construct real $\Theta$ representations. Daté [9a], using classical results on symmetric Riemann surfaces [9b], has proved existence of real $\Theta$ representations for general $N$. Dubrovin and Natanson [13] constructed real two-phase solutions in the same manner as [6,9]. Enol'skii and Belokolos [16] also constructed real two-phase solutions.

Aside from the $N=1$ case, however, the motion of the $\mu$ spectrum for real flows has remained an unsolved problem. As well, the complete real isospectral class has remained uncharacterized. Multiple components have been exhibited for $N=1,2$ $[4,6,13]$, but the proof that the list is exhaustive remains. These problems, for arbitrary $N$, are the focus of this paper. 


\section{A. Distinctions Between (sG) and $\mathrm{KdV}$, (sh-G)}

The Hill operator associated to $\mathrm{KdV}$, and the (FT) or (AKNS) operators associated to the sinh-Gordon equation (sh-G), are self-adjoint for real potentials. The simple periodic spectrum $\Sigma^{(s)}$ is entirely real. The auxiliary spectra (the $\boldsymbol{\mu}$ variables) are real and, moreover, are confined to the instability gaps on the real axis by classical spectral oscillation arguments. Thus the motion of the $\boldsymbol{\mu}$ variables is completely determined by spectral arguments, and they are bona fide coordinates for the isospectral manifold.

The associated (sG) linear eigenvalue problem, (I.2a), is nonself-adjoint for real potentials $u, w$ [11]. Thus the spectrum of (I.2a), regardless of boundary conditions, is not necessarily real. In particular, $E_{j} \in \Sigma^{(s)}$ occur either in negative pairs or complex conjugate pairs; the concept of "gaps" in $\Sigma^{(s)}$ is lost. The situation with the auxiliary spectra $\boldsymbol{\mu}(x, t)$ is much worse; for $N>1$, they no longer lie on fixed curves in $\mathscr{R}$. The spectral methods developed for $\mathrm{KdV}[14]$ and sinh-Gordon [5] simply do not apply.

However, the common feature of $N$-phase solutions for all these pde's (sG, shG, $\mathrm{KdV}, \mathrm{NLS}$ ) and every member of their hierarchy is a $\boldsymbol{\mu}$ representation in which the $x, t$ flows linearize, by the Abel-Jacobi map, on the Jacobian $\mathscr{J}(\mathscr{R})$ of a hyperelliptic curve $\mathscr{R}$. Moreover, the branch points of $\mathscr{R}$ are precisely (plus perhaps $0, \infty)$ the elements of $\Sigma^{(s)}$.

We will now show how the symmetries of $\Sigma^{(s)}$ for real potentials pick out the real flows of $\ell(x, t)$ on $\mathscr{J}(\mathscr{R})$; then the nature of the Abel-Jacobi map determines the $\boldsymbol{\mu}$ cycle structure restricted to these real flows. The last, but very crucial, step is the analysis of the integration constants $\ell_{0}$ in (I.11b). This will give real $\Theta$ function representations for the solutions and determine the complete number of real connected components associated to $\Sigma_{N}^{(s)}$.

\section{B. Basis for Real Flows in $\mathscr{J}(\mathscr{R})$-Real Phases, Wavenumbers, Frequencies}

We recall the $N$-phase simple spectrum $\Sigma^{(s)},($ I.3), and the constraints (I.5) for real potentials,

$$
\begin{gathered}
\sum_{N}^{(s)}=\left\{E_{2 j-1}, E_{2 j}, j=1, \ldots, N\right\}, \\
E_{2 j}<E_{2 j-1}<0 \quad \text { or } \quad E_{2 j}^{*}=E_{2 j-1} .
\end{gathered}
$$

There are $N+1$ distinct classes of $\Sigma_{N, k}^{(s)}$, indexed by the number $k$ of real, negative pairs, $k \in\{0, \ldots, N\}$. On the Riemann surface $\mathscr{R}$ defined by (I.9b), we fix the canonical $a_{i}, b_{i}$ cycles as indicated by Fig. 2 . The normalization constants $c_{j m}$ and period matrix columns $B_{k}$, defined in (I.10), then satisfy the following constraints (use the arguments in [6] or [9]):

In all cases:

$$
\begin{gathered}
\operatorname{Im}\left(c_{j m}\right)=0, \quad \text { for all } j m, \\
\operatorname{Re}\left(B_{i k}\right)=0, \quad i \neq k ;
\end{gathered}
$$

while

$$
\operatorname{Re}\left(B_{k k}\right)=\left\{\begin{array}{lll}
0 & \text { if } & E_{2 k}<E_{2 k-1}<0, \\
\frac{1}{2} & \text { if } & E_{2 k-1}^{*}=E_{2 k} .
\end{array}\right.
$$


Note that an unambiguous ordering of the pairs has been assigned in Fig. 2; the $k^{\text {th }}$ pair $\left(E_{2 k-1}, E_{2 k}\right)$ is linked to $a_{k}, b_{k}$ cycles and $B_{k}$.

These relations now apply to yield the $N$ reality constraints on the complex phases $\ell$ (I.11b), on $\mathscr{J}(\mathscr{R})$ :

$$
\operatorname{Re}\left(\ell(x, t)-\ell_{0}\right)=\mathbf{0} .
$$

Next we construct a useful basis for the real flows of $\ell$ on $\mathscr{J}(\mathscr{R})$. This, it will turn out, is fundamental. Clearly, $e_{1}, \ldots, e_{N}, B_{1}, \ldots, B_{N}$ form a basis of $\mathscr{J}(\mathscr{R})$ with coefficients in $\mathbb{R}$. The reality constraints (II.3) pick out exactly $N$ independent lattice elements which form a basis over $\mathbb{R}$ for the real flows of $\ell-\ell_{0}$.

All lattice elements are of the form

$$
\Lambda_{n, m}=\sum_{j=1}^{N}\left(n_{j} e_{j}+m_{j} B_{j}\right), \quad n_{j}, m_{j} \in \mathbb{Z} .
$$

Equation (II.3) introduces $N$ conditions,

$$
\operatorname{Re}\left(\sum_{j=1}^{N} n_{j} e_{j}+m_{j} B_{j}\right)=0 \text {. }
$$

From (II.2b), this reduces to

$$
n_{k}+m_{k} \operatorname{Re}\left(B_{k k}\right)=0, \quad k=1, \ldots, N .
$$

Using (II.2c), these reality constraints on the lattice basis become

$$
\left\{\begin{array}{ll}
n_{k}=0 & \text { if } \quad E_{2 k}<E_{2 k-1}<0, \\
n_{k}+\frac{1}{2} m_{k}=0 & \text { if } \quad E_{2 k}^{*}=E_{2 k-1},
\end{array} \quad k=1, \ldots, N .\right.
$$

For purposes of deducing periodicity properties of solutions, we choose the minimal $n_{k}, m_{k} \in \mathbb{Z}$ such that these relations hold. We therefore have established

Proposition II.1. For any $\Sigma_{N, k}^{(s)}$ corresponding to real potentials, a basis over $\mathbb{R}$ for the real $x, t$ flows of $\ell$ in $\mathscr{J}(\mathscr{R})$ is $\mathbf{f}_{1}, \ldots, \mathbf{f}_{N}$, where

$$
\mathbf{f}_{k}=\left\{\begin{array}{lll}
B_{k} & \text { if } & E_{2 k}<E_{2 k-1}<0, \\
e_{k}-2 B_{k} & \text { if } & E_{2 k}^{*}=E_{2 k-1} .
\end{array}\right.
$$

These "real" lattice elements are also expressible as

$$
\mathbf{f}_{k}=\left\{\begin{array}{lll}
\oint_{b_{k}} \boldsymbol{\psi} & \text { if } & E_{2 k}<E_{2 k-1}<0 \\
\oint_{a_{k}-2 b_{k}} \boldsymbol{\psi} & \text { if } & E_{2 k}^{*}=E_{2 k-1} .
\end{array}\right.
$$

Next, we expand $\ell-\ell_{0}$ in this basis over $\mathbb{R}$ :

$$
\ell-\ell_{0}=\sum_{j=1}^{N} \frac{\theta_{j}}{2 \pi} \mathbf{f}_{j}
$$

By construction, $\theta_{j}$ are real, and linear in $x$ and $t$ (since $\ell$ is). Thus, $\theta_{j}(x, t)$ as defined by (II.6a) are the $N$ real phases,

$$
\theta_{j}(x, t)=\kappa_{j} x+\omega_{j} t, \quad j=1, \ldots, N ;
$$


$\kappa_{j}$ are the spatial wavenumbers and $\omega_{j}$ are the temporal frequencies of the $N$ phase (sG) wavetrains. The explicit formula for $\boldsymbol{\kappa}, \boldsymbol{\omega}$ as functions of $\Sigma_{N, k}^{(s)}$ is easily computed from (II.6a) and the formula (I.11b) for $\ell$. Denote by $F$ the matrix with columns $\mathbf{f}_{1}, \ldots, \mathbf{f}_{N}$, and let $\mathbf{c}_{1}, \mathbf{c}_{N}$ be column vectors of normalization constants, $\left(\mathbf{c}_{j}\right)_{m}=c_{m j}$.

Proposition II.2. The real phases $\boldsymbol{\theta}$, wavenumbers $\boldsymbol{\kappa}$ and frequencies $\boldsymbol{\omega}$ of the $N$ phase potentials for $\Sigma_{N, k}^{(s)}$ are explicitly given by

$$
\begin{aligned}
& \boldsymbol{\theta}=2 \pi F^{-1}\left(\ell-\ell_{0}\right), \\
& \mathbf{\kappa}=4 \pi i F^{-1}\left(\mathbf{c}_{1}+\frac{(-1)^{N+1}}{16 \sqrt{\pi E_{j}}} \mathbf{c}_{N}\right), \\
& \boldsymbol{\omega}=4 \pi i F^{-1}\left(\mathbf{c}_{1}+\frac{(-1)^{N}}{16 \sqrt{\pi E_{j}}} \mathbf{c}_{N}\right) .
\end{aligned}
$$

Since $\mathbf{f}_{j}$ are purely imaginary and $c_{j m}$ are real, $\boldsymbol{\theta}, \boldsymbol{\kappa}, \boldsymbol{\omega}$ are clearly real.

To deduce the periodicity of these potentials with respect to the real angles or phases $\theta$, we now recall the $\Theta$ quotient representation, (I.13). The automorphic properties of $\Theta(\mathbf{z})$ are, for general lattice element $\Lambda_{\mathbf{n}, \mathbf{m}}$ in (II.4),

$$
\Theta\left(\mathbf{z}+\Lambda_{\mathbf{n}, \mathbf{m}}\right)=\exp (-i \pi[\langle B \mathbf{m}, \mathbf{m}\rangle+2(\mathbf{z}, \mathbf{m})]) \Theta(\mathbf{z}) .
$$

It follows that the effect of a general period translation in $\mathscr{J}(\mathscr{R})$,

$$
\ell \rightarrow \ell+\Lambda_{\mathrm{n}, \mathrm{m}},
$$

is to transform the theta quotient in (I.13) into

$$
\frac{\Theta\left(\ell+\Delta+\frac{1}{2}+\Lambda_{\mathrm{n}, \mathrm{m}}\right)}{\Theta\left(\ell+\Delta+\Lambda_{\mathrm{n}, \mathrm{m}}\right)}=(-1)_{1}^{\frac{N}{N} m_{j}} \frac{\Theta\left(\ell+\Delta+\frac{1}{2}\right)}{\Theta(\ell+\Delta)} .
$$

However, by Proposition II.1 the reality constraints on $\mathscr{J}(\mathscr{R})$ restrict the allowable period translations $\Lambda_{\mathrm{n}, \mathrm{m}}$ to those generated by $\mathbf{f}_{k},(\mathrm{II} .5 \mathrm{a}, \mathrm{b})$. Thus the periodicity of the potential $u(x, t)=u(\theta)$ is determined by (II.7) for each real phase $\theta_{k}$.

Proposition II.3. For $\Sigma_{N, k}^{(s)}$ in (II.1), the periodicity behavior of the $N$-phase potentials $u(\theta)$ with respect to each real phase is

$$
u\left(\theta_{1}, \ldots, \theta_{k}+2 \pi, \ldots, \theta_{N}\right)=\left\{\begin{array}{lll}
u\left(\theta_{1}, \ldots, \theta_{k}, \ldots, \theta_{N}\right) & \text { if } \quad E_{2 k}^{*}=E_{2 k-1} \\
u\left(\theta_{1}, \ldots, \theta_{k}, \ldots, \theta_{N}\right) \pm 2 \pi & \text { if } & E_{2 k}<E_{2 k-1}<0
\end{array}\right.
$$

\section{Homology of the $\boldsymbol{\mu}$ Cycles for Real Flows}

The characterization of real flows $\ell(x, t) \in \mathscr{J}(\mathscr{R})$ given above, together with the Abel-Jacobi map between $\boldsymbol{\mu}$ and $\ell$, now enables us to deduce the $\mu$-cycle structure on $(\mathscr{R} \times \ldots \times \mathscr{R}) / \sigma_{N}$.

For any fixed $\ell_{0}$ in (I.11b) that yields real $u(x, t)$ in (I.13), the component $\mathscr{M}_{\ell_{0}}$ of the real isospectral class $\mathscr{M}$ is an $N$-dimensional real torus (Proposition II.1). 
(Existence of such $\ell_{0}$ and determination of all connected components of $\mathscr{M}$ is taken up in II.D.)

A parametrization of $\mathscr{M}_{\ell_{0}}$ is provided by (II.6a):

$$
\mathscr{M}_{\ell_{0}}=S^{1} \times \ldots \times S^{1}=\left\{\left(\theta_{1}, \ldots, \theta_{N}\right) \mid \theta_{j} \in[0,2 \pi)\right\} .
$$

The coordinate curves of this parametrization

$$
\left[\theta_{1} \equiv \text { const }, \ldots, \theta_{k} \in[0,2 \pi), \ldots, \theta_{N} \equiv \text { const }\right], \quad 1 \leqq k \leqq N
$$

are $N$ independent 1-cycles on $\mathscr{M}_{\ell_{0}}$. The inverse image of each of these cycles under the Abel map is a fundamental 1-cycle on the $\boldsymbol{\mu}$-torus. In this section we express these fundamental 1 -cycles in terms of the homology basis, $\left\{a_{1}, \ldots, a_{N}, b_{1}, \ldots, b_{N}\right\}$, of $\mathscr{R}$.

The $\boldsymbol{\mu}$-torus represents an $N$ dimensional cycle in the homology group $H_{N}\left(\frac{\mathscr{R} \times \ldots \times \mathscr{R}}{\sigma_{N}}, \mathbb{Z}\right)$. Using the Künneth formula one can decompose $H_{N}(\mathscr{R} \times \ldots \times \mathscr{R}, \mathbb{Z})$ into tensor products of $H_{i}(\mathscr{R}, \mathbb{Z}), i=0,1,2$. For our purposes it suffices to observe that

$$
H_{N}(\mathscr{R} \times \ldots \times \mathscr{R}, \mathbb{Z}) \cong K \oplus L,
$$

where $L=H_{1}(\mathscr{R}, \mathbb{Z}) \otimes \ldots \otimes H_{1}(\mathscr{R}, \mathbb{Z})$ and $K$ involves all tensor products having $H_{2}(\mathscr{R}, \mathbb{Z})$ as a factor. The action of $\sigma_{N}$ on $\mathscr{R} \times \ldots \times \mathscr{R}$ lifts to homology and we have

$$
H_{N}\left(\frac{\mathscr{R} \times \ldots \times \mathscr{R}}{\sigma_{N}}, \mathbb{Z}\right) \cong K^{\sigma_{N}} \oplus L^{\sigma_{N}} .
$$

$L^{\sigma_{N}}$ consists of antisymmetric tensors in $L$. (This follows because if an $N$-cycle is the product of $N$ ordered 1-cycles then interchange of two adjacent 1-cycles changes the orientation of the $N$-cycle.) A basis for $L^{\sigma_{N}}$ consists of

$$
\left\{\ldots, a_{i_{1}} \wedge \ldots \wedge a_{i_{m}} \wedge b_{j_{1}} \wedge \ldots \wedge b_{j_{N-m}}, \ldots\right\}
$$

where $1 \leqq i_{1}<i_{2}<\ldots<i_{m} \leqq N$ and $1 \leqq j_{1}<j_{2}<\ldots<j_{N-m} \leqq N$.

The Abel map, $\Psi$, induces a map

$$
\Psi_{*}: H_{N}\left(\frac{(\mathscr{R} \times \ldots \times \mathscr{R})}{\sigma_{N}}\right) \rightarrow H_{N}(\mathscr{J}) .
$$

$\Psi_{*}$ maps $L^{\sigma_{N}} 1-1$ onto $H_{N}(\mathscr{J})$. This is because the map induced by $\Psi$ in dimension 1 ,

$$
\Psi_{*}: H_{1}\left(\left(\frac{(\mathscr{R} \times \ldots \times \mathscr{R})}{\sigma_{N}}\right)\left(=H_{1}(\mathscr{R})\right)\right) \rightarrow H_{1}(\mathscr{J}),
$$

is an isomorphism given by $\Psi_{*}\left(a_{i}\right)=e_{i}, \Psi_{*}\left(b_{i}\right)=B_{i}$, where $e_{i}, B_{i}$ represent cycles which correspond to the periods $e_{i}, B_{i}$. The $N^{\text {th }}$ exterior powers of $\left\{\ldots e_{i}, B_{i} \ldots\right\}$ freely generate the image $\Psi^{*}\left(L^{\sigma_{N}}\right)$ in (II.11). Since $\operatorname{dim} L^{\sigma_{N}}=\operatorname{dim} H_{N}(\mathscr{J})$, it follows that $\operatorname{Ker} \Psi_{*}=K^{\sigma_{N}}$. Hence

$$
\Psi_{*}: L^{\sigma_{N}} \approx H_{N}(\mathscr{J}) .
$$

We now use (II.12) to describe the $\mu$-torus, $\Psi^{-1}\left(\mathscr{M}_{\ell_{0}}\right)$, in terms of the homology of $\mathscr{R}$. 
Given $\mathscr{M}_{\ell_{0}}$ with periodic shift basis

$$
\begin{gathered}
\mathbf{f}_{1}=n_{11} e_{1}+\ldots+n_{1 N} e_{N}+m_{11} B_{1}+\ldots+m_{1 N} B_{N} \\
\vdots \\
\vdots \\
\mathbf{f}_{N}=n_{N 1} e_{1}+\ldots+n_{N N} e_{N}+m_{N 1} B_{1}+\ldots+m_{N N} B_{N}
\end{gathered}
$$

the fundamental $\boldsymbol{\mu}$-cycles are simply given by

$$
\Psi_{*}^{-1}\left(\left[\mathbf{f}_{k}\right]\right)=n_{k 1} a_{1}+n_{k 2} a_{2}+\ldots+n_{k N} a_{N}+m_{k 1} b_{1}+\ldots+m_{k N} b_{N} .
$$

Thus we easily deduce the fundamental $\mu$-cycles for $N$-phase sine-Gordon wavetrains (see II.5):

$$
\mu_{k} \text { cycle } \sim\left\{\begin{array}{lll}
b_{k} & \text { if } & E_{2 k}<E_{2 k-1}<0 \\
a_{k}-2 b_{k} & \text { if } & E_{2 k}^{*}=E_{2 k-1}
\end{array} .\right.
$$

To determine the homology of the $\mu$-torus in $H_{N}\left((\mathscr{R} \times \ldots \times \mathscr{R}) / \sigma_{N}\right)$, one simply expands the exterior product

$$
\Psi_{*}^{-1}\left(\left[\mathbf{f}_{1}\right]\right) \wedge \ldots \wedge \Psi_{*}^{-1}\left(\left[\mathbf{f}_{N}\right]\right)
$$

in the basis (II.10) by using (II.13). By (II.14) we see that in fact the $\boldsymbol{\mu}$-torus is homologus to a product of cycles $\gamma_{1} \times \ldots \times \gamma_{N}$, where $\gamma_{i}$ is a cycle on $\mathscr{R}$. For example, an $N$-kink torus has $\gamma_{1}=b_{1}, \ldots, \gamma_{N}=b_{N}$, while a breather torus has $\gamma_{1}=a_{1}$ $-2 b_{1}, \ldots, \gamma_{N}=a_{N}-2 b_{N}$. So although the sine-Gordon $\boldsymbol{\mu}$-trajectories do not lie on fixed paths as do those of $\mathrm{KdV}$, the $\boldsymbol{\mu}$-cycles do remain in a fixed homology class. This fact is fundamental for the modulation theory of $N$-phase sine-Gordon wavetrains [17].

\section{The Real Isospectral Class $\mathscr{M}$ of $\sum_{N, k}^{(s)}$}

We now determine the full real isospectral class. Any choice of $\ell_{0}$ in (I.11b) that yields real $u(x, t)$ corresponds to a real $N$-torus $\mathscr{M}_{\ell_{0}} \subset \mathscr{M}$. For such $\ell_{0}=\ell(\boldsymbol{\theta}=\mathbf{0})$, the entire real torus $\mathscr{M}_{\ell_{0}}$ is swept out as $\boldsymbol{\theta}$ varies over $[0,2 \pi) \times \ldots \times[0,2 \pi)$, and each value of $\ell(\theta)$ yields another $\ell_{0}$ in the same connected component. Therefore we define an equivalent relation on initial conditions $\ell_{0}$ :

$$
\ell_{0}^{1} \sim \ell_{0}^{2} \quad \text { if } \quad \ell_{0}^{1}-\ell_{0}^{2}=\sum_{1}^{N} \theta_{j} \mathbf{f}_{j} \bmod \Lambda
$$

The existence of real components $\mathscr{M}_{\ell_{0}}$ is easy to deduce from the theta quotient representation (I.13). $u(x, t)$ will be real-valued if

$$
\Theta\left(\ell+\Delta+\frac{1}{2}\right)=[\Theta(\ell+\Delta)]^{*} .
$$

Since $\Delta$ is a half-period, the symmetries of $\ell-\ell_{0}$ and $B$ in (II.2, 3) show $([4,6])$ that (II.15a) is equivalent to

$$
\operatorname{Re}\left[\left(\ell_{0}\right)_{k}\right]=\left\{\begin{array}{rll}
0 & \text { if } & E_{2 k}^{*}=E_{2 k-1} \\
\pm \frac{1}{4} & \text { if } & E_{2 k}<E_{2 k-1}<0 .
\end{array}\right.
$$

Since $\ell-\ell_{0} \in i \mathbb{R}^{N}$, these relations provide the existence of real components $\mathscr{M}_{\ell_{0}}$. In fact, these are exhaustive. 
Proposition II.4. Let $\Sigma_{N, k}^{(s)}$ be given by (II.1a, b), where the index $k$ denotes the number of real negative pairs, $k \in\{0, \ldots, N\}$. The real isospectral class for $\sum_{N, k}^{(s)}$ has $2^{k}$ connected components $\mathscr{M}_{\ell_{0}}$. The $2^{k}$ equivalence classes of $\ell_{0}$ are given by (II.15b).

To establish this result, we have to prove that the above constructions yield all possible $\ell_{0}$ for $\Sigma_{N, k}^{(s)}$. That is, the only way $u$ in (I.13) is real is if (II.15a) is satisfied. This is nontrivial. Our proof requires information about squared eigenfunctions for real potentials and the Kummer variety. For this reason, we defer the proof to Appendix B.

Remark 1. We encourage the reader to read this argument, however, for several reasons. First, it applies to all soliton equations connected to hyperelliptic curves (e.g., KdV, NLS, sh-G). Second, it shows how the hyperelliptic geometry of the $\mu_{j}$ variables on $\mathscr{R},\left(\mu_{j},+R\left(\mu_{j}\right)\right),\left(\mu_{j},-R\left(\mu_{j}\right)\right)$, is responsible for the possibility of two connected components in each real degree of freedom.

Remark 2. Already, this is apparent in the $N=1$ example of Sect. I.G in terms of the sheet interchange, $\mu$ cycle $\rightarrow-\mu$ cycle. From Proposition II.3 one also finds that this involution, for real pairs $E_{2 k-1}<E_{2 k}<0$, interchanges kink components and antikink components. This is apparent by tracing the involution to the real angle

$$
\theta_{k}: \mu_{k} \rightarrow-\mu_{k} \text { cycle } \Leftrightarrow \mathbf{f}_{k} \rightarrow-\mathbf{f}_{k} \Leftrightarrow \theta_{k} \rightarrow-\theta_{k} .
$$

By (II.8), this effects a switch in the "charge" of $u$ :

$$
u\left(\theta_{k}+2 \pi\right)=u \pm 2 \pi \Rightarrow u\left(-\theta_{k}+2 \pi\right)=u \mp 2 \pi .
$$

Again by Proposition II.3, for conjugate pairs $E_{2 k}^{*}=E_{2 k-1}$, the single component is invariant under the involution, $u\left(\theta_{k} \pm 2 \pi\right)=u\left(-\theta_{k} \pm 2 \pi\right)$.

Remark 3. There is another context in which the hyperelliptic involution is gainfully exploited. We have focused on the zeroes, $\boldsymbol{\mu}$, of the squared eigenfunction $g$; the zeroes of $h$, call them $v_{1}, \ldots, v_{N}$, are equivalent information. This follows from the invariance of the squared eigenfunction system (I.4) under the replacement $(f, g, h ; u, w)$ by $(f, h, g ;-u,-w)$. Thus, interchange of $\boldsymbol{\mu}$ and $\boldsymbol{v}$ variables corresponds to the involution $(u, w) \rightarrow(-u,-w)$. (This involution is also equivalent to conjugation of $\boldsymbol{\mu}$ because $g^{*}=h$ for $E>0$.) From the $\Theta$ representation of $u$, (I.13), this involution is characterized on $\mathscr{J}(\mathscr{R})$ by the half-period translation $\ell \rightarrow \ell+\frac{1}{2}$. Of course the full complex isospectral manifold is invariant under this involution. However, it follows from the structure of $\ell_{0}$, (II.15b), that translation by $\frac{1}{2}$ also leaves invariant the full real isospectral manifold of $\Sigma_{N, k}^{(s)}$, but moreover yields an involution on the real connected components: $\mathscr{M}_{\ell_{0}} \rightarrow \mathscr{M}_{-\ell_{0}}$. Each purely oscillatory degree of freedom is unaffected whereas the kink-antikink modes are interchanged. (Recall Fig. 3a, b and the Remark in Sect. I.G.)

\section{The Kummer Surface}

In the remainder of this paper we present a method for explicitly parametrizing the $\boldsymbol{\mu}$-flows in $x$ and $t$. This amounts to the inversion of the Abel-Jacobi map. Inversion formulae were known classically (see, for example, [19]) and have been reproduced 
recently in connection with soliton equations [8]. The method we present is geometric in nature and, to our knowledge, is new. The geometry enters by way of an algebraic variety, the Kummer variety, which is defined in terms of quadratic theta functions on $\mathscr{J}(\mathscr{R})$. Our derivation of the inversion formulae is based upon orthogonality relations for quadratic theta functions (Appendix B). Under the polynomial ansatz (Sect. I.D), squared eigenfunctions are quadratic theta functions. As motivation we mention that orthogonality relations for squared eigenfunctions $[11,20]$ are related to the orthogonality relations used here (III.C).

The geometry of higher dimensional Kummer varieties has not been as thoroughly treated as that of the Kummer surface. Therefore, we will restrict ourselves, in this section, to the case of 2-phases.

Our discussion will concern general Riemann surfaces of genus 2 and so will be applicable to the study of 2-phase solutions of any of the soliton equations mentioned earlier.

\section{A. Quadratic Theta Functions}

Definition. An $r^{\text {th }}$-order theta function, $\Theta(\mathbf{z})$, associated to the period matrix $B$, is a holomorphic function on $\mathbb{C}^{N}$ which satisfies the transformation formula

$$
\Theta(\mathbf{z}+\mathbf{n}+B \mathbf{m})=[\exp \{-i \pi(\langle B \mathbf{m}, \mathbf{m}\rangle+2\langle\mathbf{z}, \mathbf{m}\rangle)\}]^{r} \cdot \Theta(z),
$$

where $\boldsymbol{m}, \boldsymbol{n} \in \mathbb{Z}^{N}$.

Observe, from (II.7a), that the Riemann theta function is a first order theta function. For future reference we will denote the factor of automorphy by

$$
e_{\lambda}(\mathbf{z})=\exp \{-i \pi(\langle B \mathbf{m}, \mathbf{m}\rangle+2\langle\mathbf{z}, \mathbf{m}\rangle)\},
$$

where $\lambda=\mathbf{n}+B \mathbf{m}$.

Since $\Lambda=\left\{\mathbf{n}+B \mathbf{m} \mid \mathbf{n}, \mathbf{m} \in \mathbb{Z}^{2}\right\}$, it follows from the transformation formula (III.1) that if $f(z)=\Theta_{1}(z) / \Theta_{2}(z)$, where $\Theta_{1}, \Theta_{2}$ are $r^{\text {th }}$-order theta functions associated to $B$, then $f(z)$ is well-defined on $\mathscr{J}=\mathbb{C}^{N} / \Lambda$.

We want to consider second order (quadratic) theta functions. For fixed $r$, the set of all $r^{\text {th }}$-order theta functions is a vector space, $V_{r}$. Elementary Fourier analysis enables one to conclude that $\operatorname{dim} V_{r}=r^{N}$ [21]. Thus the space of all quadratic theta functions is $2^{N}$-dimensional.

From now on fix $N=2 . V_{2}$ is then 4-dimensional. We choose a basis of functions for $V_{2}$ as follows:

$$
\begin{aligned}
x(\mathbf{z}) & =\Theta\left[\begin{array}{ll}
0 & 0 \\
0 & 0
\end{array}\right](2 \mathbf{z} ; 2 B)=\sum_{\mathbf{m} \in \mathbb{Z}^{2}} \exp 2 \pi i\{\langle B \mathbf{m}, \mathbf{m}\rangle+2\langle\mathbf{m}, \mathbf{z}\rangle\}, \\
y(\mathbf{z}) & =\Theta\left[\begin{array}{ll}
1 & 0 \\
0 & 0
\end{array}\right](2 \mathbf{z} ; 2 B) \\
& =\sum_{\mathbf{m} \in \mathbb{Z}^{2}} \exp 2 \pi i\left\{\left\langle B\left(\mathbf{m}+\left(\frac{1}{2}, 0\right)^{T}\right), \mathbf{m}+\left(\frac{1}{2}, 0\right)^{T}\right\rangle+2\left\langle\mathbf{m}+\left(\frac{1}{2}, 0\right)^{T}, \mathbf{z}\right\rangle\right\}, \\
z(\mathbf{z}) & =\Theta\left[\begin{array}{ll}
0 & 1 \\
0 & 0
\end{array}\right](2 \mathbf{z} ; 2 B) \\
& =\sum_{\mathbf{m} \in \mathbb{Z}^{2}} \exp 2 \pi i\left\{\left\langle B\left(\mathbf{m}+\left(0, \frac{1}{2}\right)^{T}\right), \mathbf{m}+\left(0, \frac{1}{2}\right)^{T}\right\rangle+2\left\langle\mathbf{m}+\left(0, \frac{1}{2}\right)^{T}, \mathbf{z}\right\rangle\right\},
\end{aligned}
$$




$$
\begin{aligned}
w(\mathbf{z}) & =\Theta\left[\begin{array}{ll}
1 & 1 \\
0 & 0
\end{array}\right](2 \mathbf{z} ; 2 B) \\
& =\sum_{\mathbf{m} \in \mathbb{Z}^{2}} \exp 2 \pi i\left\{\left\langle B\left(\mathbf{m}+\left(\frac{1}{2}, \frac{1}{2}\right)^{T}\right), \mathbf{m}+\left(\frac{1}{2}, \frac{1}{2}\right)^{T}\right\rangle+2\left\langle\mathbf{m}+\left(\frac{1}{2}, \frac{1}{2}\right)^{T}, \mathbf{z}\right\rangle\right\} .
\end{aligned}
$$

Using the $V_{2}$-basis, (III.2), we define a natural map of $\mathscr{J}$ into $\mathbb{P}^{3}$ as follows:

$$
\begin{gathered}
\Phi: \mathscr{J} \rightarrow \mathbb{P}^{3}, \\
\mathbf{z} \rightarrow[x(\mathbf{z}): y(\mathbf{z}): z(\mathbf{z}): w(\mathbf{z})] .
\end{gathered}
$$

$\Phi$ is well-defined on $\mathscr{J}$ because if $\lambda \in \Lambda$,

$$
\begin{aligned}
& {[x(\mathbf{z}+\lambda): y(\mathbf{z}+\lambda): z(\mathbf{z}+\lambda): w(\mathbf{z}+\lambda)]} \\
& \quad=\left[e_{\lambda}^{2}(\mathbf{z}) x(\mathbf{z}): e_{\lambda}^{2}(\mathbf{z}) y(\mathbf{z}): e_{\lambda}^{2}(\mathbf{z}) z(\mathbf{z}): e_{\lambda}^{2}(\mathbf{z}) w(\mathbf{z})\right] \\
& \quad=[x(\mathbf{z}): y(\mathbf{z}): z(\mathbf{z}): w(\mathbf{z})]
\end{aligned}
$$

by homogeneity of these coordinates.

This map was classically well known, and we will state here some of its salient features. Details may be found in [22,23].

1. The image of $\Phi$ is an algebraic surface, $S$, defined by a homogeneous polynomial, $F$, of degree 4 . (We describe this polynomial below.) $S$ is referred to as a Kummer surface.

2. $\Phi$ is a, generically, $2: 1$ map of $\mathscr{J}$ onto its image. [Because $x, y, z, w$ are all even functions, $\Phi(\mathbf{z})=\Phi(-\mathbf{z})$.]

$\mathbb{C}^{2}$ acts on $\mathscr{J}$ by translations. The translations, $T_{\mathbf{z}_{0}}: \mathbf{z} \rightarrow \mathbf{z}+\mathbf{z}_{0}$, induce linear automorphisms of the space of holomorphic functions on $\mathbb{C}^{2}$ by

$$
T_{\mathbf{z}_{0}} \cdot f(\mathbf{z})=f\left(\mathbf{z}+\mathbf{z}_{0}\right) .
$$

For general $\mathbf{z}_{0}, T_{\mathbf{z}_{0}}$ does not preserve the space of $r^{\text {th }}$-order theta functions.

\begin{tabular}{|c|c|c|}
\hline generators & $\begin{array}{c}\text { 1/2-period } \\
\text { translation of } \mathscr{J}\end{array}$ & $\begin{array}{c}\text { projective } \\
\text { automorphism of } S\end{array}$ \\
\hline$\varphi_{1}$ & $z \mapsto z+\frac{e_{1}}{2}$ & {$\left[\begin{array}{rrrr}1 & 0 & 0 & 0 \\
0 & -1 & 0 & 0 \\
0 & 0 & 1 & 0 \\
0 & 0 & 0 & -1\end{array}\right]$} \\
\hline$\varphi_{2}$ & $z \mapsto z+\frac{B_{1}}{2}$ & {$\left[\begin{array}{llll}0 & 1 & 0 & 0 \\
1 & 0 & 0 & 0 \\
0 & 0 & 0 & 1 \\
0 & 0 & 1 & 0\end{array}\right]$} \\
\hline
\end{tabular}
However, the group of half period translations, $\left(\frac{1}{2} \Lambda\right) / \Lambda$, preserves the space of quadratic theta functions. This is a group of order 16 which is of central importance in passing from analytic objects on $\mathscr{J}$ to algebraic ones on $S$.

The table below exhibits generators of $\left(\frac{1}{2} \Lambda\right) / \Lambda$, the translation each generator represents, and the induced projective linear transformation on $V_{2}$ with respect to the basis $x, y, z, w$. (By projective we mean that the actual linear transformation differs possibly by an overall scalar function of $z$.) 


$$
\begin{array}{cc}
\varphi_{3} \quad z \mapsto z+\frac{e_{2}}{2} & {\left[\begin{array}{rrrr}
1 & 0 & 0 & 0 \\
0 & 1 & 0 & 0 \\
0 & 0 & -1 & 0 \\
0 & 0 & 0 & -1
\end{array}\right]} \\
\varphi_{4} \quad & {\left[\begin{array}{llll}
0 & 0 & 1 & 0 \\
0 & 0 & 0 & 1 \\
1 & 0 & 0 & 0 \\
0 & 1 & 0 & 0
\end{array}\right]}
\end{array}
$$

Since the half-period translations map $\mathscr{J}$ onto itself, it follows that the induced projective automorphisms must map the Kummer surface $S$ onto itself. Moreover, it can be shown that the group generated by $\left\{\varphi_{1}, \varphi_{2}, \varphi_{3}, \varphi_{4}\right\}$ are the only projective automorphisms of $S$ [22]. Therefore, the polynomial of degree 4 in $x, y, z, w$ which defines $S$ must be invariant under these automorphisms. The most general degree 4 polynomial satisfying this is of the form

$$
\begin{aligned}
F(x, y, z, w)= & x^{4}+y^{4}+z^{4}+w^{4}+A\left(x^{2} w^{2}+y^{2} z^{2}\right) \\
& +B\left(y^{2} w^{2}+z^{2} x^{2}\right)+C\left(z^{2} w^{2}+x^{2} y^{2}\right)+2 D x y z w \\
= & 0 .
\end{aligned}
$$

The coefficients $A, B, C, D$ are determined by the $B$-matrix. We will describe this in the next part of this section.

\section{B. Nodes and Tropes}

We observe that the second order theta functions are all even. Hence $\Phi(-\mathbf{z})=\Phi(\mathbf{z})$. In fact, as we mentioned before, $\Phi$ is a generically $2: 1$ map of $\mathscr{J}$ onto $S$ such that the points $\mathbf{z}$ and $-\mathbf{z}$ get identified. However, when $\mathbf{z} \equiv-\mathbf{z} \bmod \Lambda$ the map $\Phi$ fails to be 2:1 and therefore the image of such a point is a singularity of $S$. Elsewhere $S$ is smooth. These singularities are called nodes.

Definition. A singularity of an algebraic surface in $\mathbb{P}^{3}$ is called a node if the first nonvanishing term of the Taylor expansion of $F$ (the defining polynomial) is quadratic.

Observe that $\mathbf{z} \equiv-\mathbf{z} \bmod \Lambda$ iff $\mathbf{z}$ is a half-period. Therefore there are 16 nodes on $S$ and each is the image of a half-period in $\mathscr{J}$. Since the group of half-periods acts transitively on itself, it follows that the projective automorphisms of $S$ act transitively on the nodes; i.e., given any two nodes, there is a symmetry of $S$ carrying one node to the other.

Let us consider the node corresponding to the half-period $\mathbf{z}=0$.

Definition. A theta null is the value of a theta function at $\mathbf{z}=0$.

From our basis of quadratic theta functions we get four theta nulls which we denote as follows:

$$
\begin{array}{ll}
\alpha=\Theta_{2 B}\left[\begin{array}{ll}
0 & 0 \\
0 & 0
\end{array}\right](0), & \beta=\Theta_{2 B}\left[\begin{array}{ll}
1 & 0 \\
0 & 0
\end{array}\right](0), \\
\gamma=\Theta_{2 B}\left[\begin{array}{ll}
0 & 1 \\
0 & 0
\end{array}\right](0), & \delta=\Theta_{2 B}\left[\begin{array}{ll}
1 & 1 \\
0 & 0
\end{array}\right](0) .
\end{array}
$$


In this notation, the node corresponding to the half-period $\mathbf{z}=0$ has projective coordinates $[\alpha: \beta: \gamma: \delta]$. Also, the coefficients of the Kummer equation (III.2) are expressible in terms of theta nulls [22]:

$$
\begin{aligned}
A= & \frac{\beta^{4}+\gamma^{4}-\alpha^{4}-\delta^{4}}{\alpha^{2} \delta^{2}-\beta^{2} \gamma^{2}}, \\
B= & \frac{\gamma^{4}+\alpha^{4}-\beta^{4}-\delta^{4}}{\beta^{2} \delta^{2}-\gamma^{2} \alpha^{2}}, \\
C= & \frac{\alpha^{4}+\beta^{4}-\gamma^{4}-\delta^{4}}{\gamma^{2} \delta^{2}-\alpha^{2} \beta^{2}}, \\
D= & \frac{\alpha \beta \gamma \delta\left(\delta^{2}+\alpha^{2}-\beta^{2}-\gamma^{2}\right)\left(\delta^{2}+\beta^{2}-\gamma^{2}-\alpha^{2}\right)}{\left(\alpha^{2} \delta^{2}-\beta^{2} \gamma^{2}\right)\left(\beta^{2} \delta^{2}-\gamma^{2} \alpha^{2}\right)} \\
& \frac{\cdot\left(\delta^{2}+\gamma^{2}-\alpha^{2}-\beta^{2}\right)\left(\alpha^{2}+\beta^{2}+\gamma^{2}+\delta^{2}\right)}{\left(\gamma^{2} \delta^{2}-\alpha^{2} \beta^{2}\right)} .
\end{aligned}
$$

The nodes of $S$ are arrayed in a remarkable configuration:

Definition. A trope is a plane in $\mathbb{P}^{3}$ which contains exactly 6 nodes of the Kummer surface $S$.

The importance of tropes lies in their relation to quadratic theta functions. The intersection of a trope with $S$ is a curve, $\Theta$, contained in $S$. $\Theta$ is the image, under $\Phi: \mathscr{J} \rightarrow S$, of the zero locus of a special second order theta function.

It is classically known [22] that there are exactly 16 tropes. We will list all the tropes below. In an appropriate sense, a trope is dual to a node. To be precise, a trope is a plane which is tangent to $S$ along a conic (a plane curve of degree 2). This conic of contact is called a singular conic. We will sometimes abuse notation and call a singular conic a trope.

Just as each trope contains 6 nodes, each node is contained by exactly 6 tropes. This array of nodes is called the 16:6-configuration.

$[\alpha: \beta: \gamma: \delta]$ is a node (defined earlier). Associated to this node is the trope defined by

$$
\alpha x+\beta y+\gamma z+\delta w=0 .
$$

We list the six nodes contained in this trope:

$$
\begin{aligned}
& {[\delta:-\gamma: \beta:-\alpha]=\varphi_{1} \circ \varphi_{2} \circ \varphi_{4}[\alpha: \beta: \gamma: \delta],} \\
& {[\delta: \gamma:-\beta:-\alpha]=\varphi_{2} \circ \varphi_{3} \circ \varphi_{4}[\alpha: \beta: \gamma: \delta],} \\
& {[\gamma: \delta:-\alpha:-\beta]=\varphi_{3} \circ \varphi_{4}[\alpha: \beta: \gamma: \delta],} \\
& {[-\gamma: \delta: \alpha:-\beta]=\varphi_{1} \circ \varphi_{3} \circ \varphi_{4}[\alpha: \beta: \gamma: \delta],} \\
& {[-\beta: \alpha: \delta:-\gamma]=\varphi_{1} \circ \varphi_{2} \circ \varphi_{3}[\alpha: \beta: \gamma: \delta],} \\
& {[\beta:-\alpha: \delta:-\gamma]=\varphi_{1} \circ \varphi_{2}[\alpha: \beta: \gamma: \delta] .}
\end{aligned}
$$

The projective automorphisms of $S$ carry tropes to tropes. Hence the other 15 tropes and the nodes which they contain can be determined from the above trope by letting the group of 16 projective automorphisms of $S$ act on $\mathbb{P}^{3}$. 


\section{The Orthogonal Matrix of Tropes}

We shall now introduce a compact notation for the tropes which will make them easier to deal with in computations and which will also clarify the structure of our arguments later on.

The projective automorphisms introduced in III.A can be decomposed into two types: permutations, generated by $\varphi_{2}$ and $\varphi_{4}$, and changes of sign, generated by $\varphi_{1}$ and $\varphi_{3}$.

We label elements of the subgroup of permutations by

$$
\begin{aligned}
& d=\text { identity, } \\
& c=\varphi_{2}, \\
& b=\varphi_{4}, \\
& a=\varphi_{2} \circ \varphi_{4} .
\end{aligned}
$$

In parallel, we label the elements of the subgroup of sign changes by

$$
\begin{aligned}
& d=\text { identity, } \\
& c=-\varphi_{3}, \\
& b=-\varphi_{1}, \\
& a=\varphi_{1} \circ \varphi_{3} .
\end{aligned}
$$

This seemingly curious choice of labels is made to keep consistent with classical notation (see [22]). We can now label each trope by the ordered pair, (permutation, sign change), corresponding to the projective automorphism of $S$ which transforms the linear form defining the trope into $\alpha x+\beta y+\gamma z+\delta w$. For example,

$$
\begin{aligned}
& (d d)=(\alpha, \beta, \gamma, \delta) \cdot(x, y, z, w)=\alpha x+\beta y+\gamma z+\delta w, \\
& (b c)=(\gamma, \delta, \alpha, \beta) \cdot(-x,-y, z, w)=-\gamma x-\delta y+\alpha z+\beta w .
\end{aligned}
$$

The linear forms of these tropes are connected by a remarkable set of algebraic identities whose geometric interpretation will be important for us. Observe that if we assume that $\alpha^{2}+\beta^{2}+\gamma^{2}+\delta^{2}=1$ and $x^{2}+y^{2}+z^{2}+w^{2}=1$ then the matrices

$$
\left[\begin{array}{rrrr}
\alpha & \beta & \gamma & \delta \\
\delta & -\gamma & \beta & -\alpha \\
\gamma & \delta & -\alpha & -\beta \\
-\beta & \alpha & \delta & -\gamma
\end{array}\right] \text { and }\left[\begin{array}{rrrr}
x & w & -z & y \\
y & z & w & -x \\
z & -y & x & w \\
w & -x & -y & -z
\end{array}\right]
$$

are orthogonal and, hence, their product is an orthogonal matrix (i.e., the column vectors are orthonormal). The normality assumptions we made are of no consequence since we shall always be working in homogeneous coordinates. The product of these two matrices yields an orthogonal matrix of tropes:

$$
\left[\begin{array}{rrrr}
(d d) & (a c) & (b a) & (c b) \\
-(a b) & (d a) & -(c c) & (b d) \\
-(b c) & (c d) & (d b) & -(a a) \\
-(c a) & -(b b) & (a d) & (d c)
\end{array}\right]
$$


On substituting $x=\alpha, y=\beta, z=\gamma, w=\delta$ into these linear forms, six of them vanish and the other ten become quadratic expressions in the theta nulls which we will refer to as the ten fundamental quadrics. We will denote the fundamental quadric obtained through this substitution by square brackets "[ ]".

Thus we obtain an orthogonal matrix of fundamental quadrics:

$$
\begin{gathered}
{\left[\begin{array}{cccc}
{[d d]} & 0 & 0 & 0 \\
0 & {[d a]} & -[c c] & {[b d]} \\
0 & {[c d]} & {[d b]} & -[a a] \\
0 & -[b b] & {[a d]} & {[d c]}
\end{array}\right]} \\
=\left[\begin{array}{cccc}
\alpha^{2}+\beta^{2}+\gamma^{2}+\delta^{2} & 0 & 0 & 0 \\
0 & \alpha^{2}-\beta^{2}-\gamma^{2}+\delta^{2} & 2 \alpha \beta-2 \gamma \delta & 2 \gamma \alpha+2 \beta \delta \\
0 & 2 \alpha \beta+2 \gamma \delta & -\alpha^{2}+\beta^{2}-\gamma^{2}+\delta^{2} & 2 \beta \gamma-2 \alpha \delta \\
0 & 2 \gamma \alpha-2 \beta \delta & 2 \beta \gamma+2 \alpha \delta & -\alpha^{2}-\beta^{2}+\gamma^{2}+\delta^{2}
\end{array}\right] .
\end{gathered}
$$

The observation that will be most important for our computations is that the lower right $3 \times 3$ matrix is orthogonal when all entries are divided by $[d d]=\alpha^{2}+\beta^{2}$ $+\gamma^{2}+\delta^{2}$. This implies a variety of quadratic relations between the fundamental quadrics.

\section{Jacobi Inversion}

In this part we introduce the Abel-Jacobi map from the Riemann surface $\mathscr{R}$, where each of the $\mu$-coordinates lives, into the Jacobian $\mathscr{J}$ where the phases $\ell(x, t)$ reside. We then develop a connection between the branch points of $\mathscr{R}$ and the nodes of $S$. Finally we discuss the solution of the Jacobi inversion problem.

Let $p_{0}, p_{1}$ denote two points on $\mathscr{R}$; in general we will regard $p_{0}$ as fixed and $p_{1}$ as variable. Let $\psi_{1}, \psi_{2}$ be the normalized holomorphic differentials defined in Sect. I. We introduce the notation

$$
\left[p_{1}\right]-\left[p_{0}\right]=\left\langle\int_{p_{0}}^{p_{1}} \psi_{1}, \int_{p_{0}}^{p_{1}} \psi_{2}\right\rangle^{T}
$$

in terms of which the Abel-Jacobi map $\Psi: \mathscr{R} \times \mathscr{R} / \sigma_{2} \rightarrow \mathscr{J}$ is

$$
\left(p_{1}, p_{2}\right) \rightarrow\left[p_{1}\right]+\left[p_{2}\right]-2\left[p_{0}\right]=\left\langle\int_{p_{0}}^{p_{1}} \psi_{1}+\int_{p_{0}}^{p_{2}} \psi_{1}, \int_{p_{0}}^{p_{1}} \psi_{2}+\int_{p_{0}}^{p_{2}} \psi_{2}\right\rangle^{T} \text {. }
$$

We refer the reader to $[12,13]$ for further discussions of this important map. In succeeding sections, the fundamental fact to bear in mind is that when the unordered pair $\left(p_{1}, p_{2}\right)=\left(\mu_{1}(x, t), \mu_{2}(x, t)\right)$, then its image in $\mathscr{J}$ under the AbelJacobi map is the phase vector $\ell(x, t)$ :

$$
\ell(x, t)=\left[\mu_{1}(x, t)\right]+\left[\mu_{2}(x, t)\right]-2\left[p_{0}\right] .
$$

\section{E. Branch Points and Nodes}

From now on we will set $p_{0}=\infty$. (This choice in no way affects the reality constraints on the $\boldsymbol{\mu}$-coordinates; it merely fixes an origin in $\mathscr{J}$.)

$$
\begin{gathered}
\mathscr{R} \rightarrow \mathscr{J} \\
\left(p_{1}, \infty\right) \rightarrow\left[p_{1}\right]+[\infty]-2[\infty]=\left[p_{1}\right]-[\infty] .
\end{gathered}
$$


Using intersection theory (p. 49 of [23]) one can determine the images in $\mathscr{J}$ of the branch points of $\mathscr{R}$. These are all half-periods, and so they map, under the theta map $\Phi$, to nodes on $S$. Below, we list these correspondences. For future reference we also give the projective automorphisms corresponding to these six nodes.

Table 1

\begin{tabular}{llll}
\hline & $\begin{array}{l}v_{i} \\
\text { (half period) }\end{array}$ & $\begin{array}{l}\Phi\left(v_{i}\right) \\
\text { (node) }\end{array}$ & $\begin{array}{l}\text { (Projective } \\
\text { automorphism of } S \text { ) }\end{array}$ \\
\hline$v_{0}=\left[E_{1}\right]-[\infty]$ & $\left(e_{1}+e_{2}+B_{1}\right) / 2$ & {$[\beta:-\alpha:-\delta: \gamma]$} & $\varphi_{1} \circ \varphi_{2} \circ \varphi_{3}$ \\
$v_{1}=\left[E_{2}\right]-[\infty]$ & $\left(e_{2}+B_{1}\right) / 2$ & {$[\beta: \alpha:-\delta:-\gamma]$} & $\varphi_{2} \circ \varphi_{3}$ \\
$v_{2}=[0]-[\infty]$ & $\left(e_{1}+e_{2}\right) / 2$ & {$[\alpha:-\beta:-\gamma: \delta]$} & $\varphi_{1} \circ \varphi_{3}$ \\
$v_{3}=[\infty]-[\infty]$ & 0 & {$[\alpha: \beta: \gamma: \delta]$} & Id \\
$v_{4}=\left[E_{3}\right]-[\infty]$ & $\left(e_{2}+B_{2}\right) / 2$ & {$[\gamma: \delta:-\alpha:-\beta]$} & $\varphi_{3} \circ \varphi_{4}$ \\
$v_{5}=\left[E_{4}\right]-[\infty]$ & $B_{2} / 2$ & {$[\gamma: \delta: \alpha: \beta]$} & $\varphi_{4}$ \\
\hline
\end{tabular}

The nodes corresponding to $v_{0}, \ldots, v_{5}$ lie on a unique trope and are, in fact, the six nodes of that trope. To see this one simply observes that these nodes satisfy the linear equation of the trope

$$
-(a b): \delta x-\gamma y+\beta z-\alpha w=0 .
$$

This trope is the image of $\mathscr{R} \times\{\infty\}$ under the map $\Phi \circ \Psi$. In particular, observe that $\Phi \circ \Psi$ is a $2: 1$ map of $\mathscr{R}$ onto the singular conic determined by $-(a b)$ (recall this terminology from III.B). This map is branched at the six nodes on this conic. In other words, $\Phi \circ \Psi$ represents $\mathscr{R}$ as a hyperelliptic curve. We will use this fact in part $F$ of this section to express the branch points in terms of fundamental quadrics.

The preimages in $\mathscr{R} \times \mathscr{R} / \sigma_{2}$ of the remaining ten nodes are given by the following table. We define

$$
v_{i j}=v_{i}+v_{j} \text { for } i, j>0, \quad i \neq j .
$$

Table 2

\begin{tabular}{llll}
\hline & Half periods & Nodes & $\begin{array}{l}\text { Projective } \\
\text { automorphism }\end{array}$ \\
\hline$v_{12}=[0]+\left[E_{2}\right]-2[\infty]$ & $e_{2} / 2$ & {$[\alpha: \beta:-\gamma:-\delta]$} & $\varphi_{3}$ \\
$v_{13}=[\infty]+\left[E_{2}\right]-2[\infty]$ & $e_{1} / 2$ & {$[\alpha:-\beta: \gamma:-\delta]$} & $\varphi_{1}$ \\
$v_{14}=\left[E_{3}\right]+\left[E_{2}\right]-2[\infty]$ & $\left(e_{1}+e_{2}+B_{2}\right) / 2$ & {$[-\gamma: \delta: \alpha:-\beta]$} & $\varphi_{1} \circ \varphi_{3} \circ \varphi_{4}$ \\
$v_{15}=\left[E_{4}\right]+\left[E_{2}\right]-2[\infty]$ & $\left(e_{1}+B_{2}\right) / 2$ & {$[\gamma:-\delta: \alpha:-\beta]$} & $\varphi_{1} \circ \varphi_{4}$ \\
$v_{23}=[\infty]+[0]-2[\infty]$ & $B_{1} / 2$ & {$[\beta: \alpha: \delta: \gamma]$} & $\varphi_{2}$ \\
$v_{24}=\left[E_{3}\right]+[0]-2[\infty]$ & $\left(e_{2}+B_{1}+B_{2}\right) / 2$ & {$[\delta: \gamma:-\beta:-\alpha]$} & $\varphi_{2} \circ \varphi_{3} \circ \varphi_{4}$ \\
$v_{25}=\left[E_{4}\right]+[0]-2[\infty]$ & $\left(B_{1}+B_{2}\right) / 2$ & {$[\delta: \gamma: \beta: \alpha]$} & $\varphi_{2} \circ \varphi_{4}$ \\
$v_{34}=\left[E_{3}\right]+[\infty]-2[\infty]$ & $\left(e_{1}+B_{1}+B_{2}\right) / 2$ & {$[\delta:-\gamma: \beta:-\alpha]$} & $\varphi_{1} \circ \varphi_{2} \circ \varphi_{4}$ \\
$v_{35}=\left[E_{4}\right]+[\infty]-2[\infty]$ & $\left(e_{1}+e_{2}+B_{1}+B_{2}\right) / 2$ & {$[\delta:-\gamma:-\beta: \alpha]$} & $\varphi_{1} \circ \varphi_{2} \circ \varphi_{3} \circ \varphi_{4}$ \\
$v_{45}=\left[E_{4}\right]+\left[E_{3}\right]-2[\infty]$ & $\left(e_{1}+B_{1}\right) / 2$ & {$[\beta:-\alpha: \delta:-\gamma]$} & $\varphi_{1} \circ \varphi_{2}$ \\
\hline
\end{tabular}




\section{F. Solution of the Jacobi Inversion Problem}

We now turn to the formulation of the primary tool in this paper. From (I.11), the Abel-Jacobi transform relates the $\mu$-coordinates to the phases $\ell$. In Sect. III we characterized the manifolds of isospectral two-phase wavetrains in terms of the phases $\ell$. Our goal is to characterize these manifolds in terms of the $\boldsymbol{\mu}$-coordinates. We will achieve this goal by inverting the Abel-Jacobi map to express the $\boldsymbol{\mu}$-coordinates as functions of $\ell$. Precisely, one derives the

Inversion Formulae

$$
\begin{gathered}
\mu_{1} \mu_{2}=\sqrt{E_{1} E_{2} E_{3} E_{4}} \frac{(a c)}{(a b)}, \\
\mu_{1}+\mu_{2}=\frac{[d a]\left\{E_{2}+E_{4}\right\}(a c)-[c c] E_{4}(b a)+[b d] E_{2}(c b)}{[d d](a b)} .
\end{gathered}
$$

In the appendix we give a geometric derivation of these formulae. In this section we will present these formulae but concentrate on a derivation (different from the one given in the appendix) of (III.13a). This formula relates the $\boldsymbol{\mu}$-representation (I.7) to the theta quotient representation (I.13).

To begin with, we observe that $\Theta^{2}(\mathbf{z})$, where $\Theta(\mathbf{z})$ is the Riemann theta function, is a quadratic theta function by definition. Hence, it can be expanded in our basis for $V_{2}$ (III.2). The result is

$$
\Theta^{2}(\mathbf{z})=\alpha x+\beta y+\gamma z+\delta w=(d d) .
$$

This expansion is most easily derived using standard addition formulae for theta functions $[12,22]$.

Next we consider $\mu_{1} \mu_{2}$ which is a symmetric function of the $\boldsymbol{\mu}$-coordinates and therefore defines a function on $\mathscr{R} \times \mathscr{R} / \sigma_{2}$. According to Siegel [12], any such function can be represented as a quotient of theta functions; and, in particular,

$$
\mu_{1} \mu_{2}=\kappa \frac{\Theta^{2}\left(\left[\mu_{1}\right]+\left[\mu_{2}\right]-2[\infty]-([0]-[\infty])-\Delta\right)}{\Theta^{2}\left(\left[\mu_{1}\right]+\left[\mu_{2}\right]-2[\infty]-\Delta\right)} .
$$

In (III.15), $\kappa$ is a multiplicative constant, which we will determine, and $\Delta \in \mathscr{J}$ is Riemann's constant which we will determine now. We recall [12].

Riemann's Theorem. If $\Theta$ denotes the zero locus of Riemann's theta function, $\Theta(\mathbf{z})$, in $\mathscr{J}$, then

$$
\Theta=\Psi(\mathscr{R} \times\{\infty\})+\Delta .
$$

From (III.14) we deduce that $\Phi(\Theta)=(d d)$, and from (III.12) we deduce that $\Phi \circ \Psi(\mathscr{R} \times\{\infty\})=-(a b)$. Since these are both tropes, it follows from (III.B) that $\Delta$ is a half-period which corresponds to the projective automorphism - $(a b)$ $=\varphi_{1} \circ \varphi_{2} \circ \varphi_{4}$. From Table 2 , we observe that the half-period corresponding to $\varphi_{1} \circ \varphi_{2} \circ \varphi_{4}$ is $\left(e_{1}+B_{1}+B_{2}\right) / 2$. Hence,

$$
\Delta=\left(e_{1}+B_{1}+B_{2}\right) / 2 .
$$


From Table 1, we now note that $[0]-[\infty]=\left(e_{1}+e_{2}\right) / 2$. Substituting into (III.15) we get

$$
\mu_{1} \mu_{2}=\kappa \frac{\Theta^{2}\left(\left[\mu_{1}\right]+\left[\mu_{2}\right]-2[\infty]+\Delta+\frac{e_{1}+e_{2}}{2}\right)}{\Theta^{2}\left(\left[\mu_{1}\right]+\left[\mu_{2}\right]-2[\infty]+\Delta\right)}
$$

When $\mu_{j}=\mu_{j}(x, t)$, we see from (III.10) that $\ell(x, t)=\left[\mu_{1}\right]+\left[\mu_{2}\right]-2[\infty]$, and thus (III.18) is the theta quotient representation which we discussed in Sect. 1. (Compare (III.18) with (I.7) and (I.13); note that $\frac{e_{1}+e_{2}}{2}=\frac{\mathbf{1}}{\mathbf{2}}$ in our earlier notation.) Equation(III.18) represents $\mu_{1} \mu_{2}$ as a ratio of quadratic theta functions and therefore $\mu_{1} \mu_{2}$ is a ratio of tropes. To determine these tropes we use the fact that $\Theta^{2}\left[\mathbf{z}+\frac{e_{1}+e_{2}}{2}\right]=\varphi_{1} \circ \varphi_{3}\left(\Theta^{2}(\mathbf{z})\right)$ since $\varphi_{1} \circ \varphi_{3}$ corresponds to $\frac{e_{1}+e_{2}}{2}$. Thus

$$
\begin{aligned}
\mu_{1} \mu_{2} & =\kappa \frac{\varphi_{1} \circ \varphi_{3}\left(\Theta^{2}(\ell+\Delta)\right)}{\Theta^{2}(\ell+\Delta)} \\
& =\kappa \frac{\varphi_{1} \circ \varphi_{3}(\alpha x+\beta y+\gamma z+\delta w)}{\alpha x+\beta y+\gamma z+\delta w} \quad \text { [by (III.14)] } \\
& =\kappa \frac{\alpha x-\beta y-\gamma z+\delta w}{\alpha x+\beta y+\gamma z+\delta w},
\end{aligned}
$$

where $x, y, z, w$ are functions of $\ell+\Delta$. We would like to regard $x, y, z, w$ as functions of just $\ell$. In this case

$$
\begin{aligned}
\mu_{1} \mu_{2} & =\kappa \frac{\varphi_{1} \circ \varphi_{2} \circ \varphi_{4}(\alpha x-\beta y-\gamma z+\delta w)}{\varphi_{1} \circ \varphi_{2} \circ \varphi_{4}(\alpha x+\beta y+\gamma z+\delta w)} \\
& =\kappa \frac{\delta x+\gamma y-\beta z-\alpha w}{\delta x-\gamma y+\beta z-\alpha w},
\end{aligned}
$$

where $x, y, z, w$ are now functions of $\ell$.

In III.E we indicated that the branch points of $\mathscr{R}$ were expressible in terms of fundamental quadrics. These expressions are derived in Appendix A, but we record them here for convenience:

$$
\begin{gathered}
E_{1}=k \frac{[d a][d b]}{[a d]}, \quad E_{2}=k \frac{[d c][d d]}{[a d]}, \quad 0=0, \\
\infty=\infty, \quad E_{3}=k \frac{[d a][d c]}{[a a]}, \quad E_{4}=k \frac{[d b][d d]}{[a a]} .
\end{gathered}
$$

( $k$ is any non-zero complex number; bear in mind that the $\mu$-coordinate, and hence the $E_{j}$, are uniquely defined only up to a linear fractional transformation.)

We will now determine the multiplicative constant $\kappa$ in (III.19). To this end set $\mu_{1}=E_{1}, \mu_{2}=E_{4}$. Then

$$
\ell(\boldsymbol{\mu})=\left[E_{1}\right]+\left[E_{4}\right]-2[\infty]
$$


From Table 1 we see that

$$
\Phi(\ell(\mu))=[\delta:-\gamma:-\beta: \alpha] .
$$

Substituting into (III.19), we get

From (III.20) we have

$$
E_{1} E_{4}=\kappa \frac{\delta^{2}-\gamma^{2}+\beta^{2}-\alpha^{2}}{\delta^{2}+\gamma^{2}-\beta^{2}-\alpha^{2}} .
$$

$$
k \frac{[d a][d b]}{[a d]} \cdot k \frac{[d b][d d]}{[a a]}=\kappa \frac{[d b]}{[d c]} .
$$

Hence,

$$
\kappa=k^{2} \frac{[d a][d b][d c][d d]}{[a a][a d]}=\sqrt{E_{1} E_{2} E_{3} E_{4}} .
$$

Putting this value we have our first formula:

$$
\mu_{1} \mu_{2}=\sqrt{E_{1} E_{2} E_{3} E_{4}} \frac{(a c)}{(a b)} .
$$

Note that the constant $\kappa$ is consistent with the $\boldsymbol{\mu}$-representation (I.7).

\section{The Real Kummer Surface}

In this section we will lay the groundwork for applying the machinery of Sect. III to the study of the global behavior of the $\mu$-variables for real 2 phase (sG) wavetrains. As earlier, $\mathscr{M}_{\ell_{0}}$ denotes a connected component of the full real isospectral class $\mathscr{M}$, $\mathscr{M}=\bigcup_{\ell_{0}} \mathscr{M}_{\ell_{0}}$. The situation can be summarized by the following diagram:

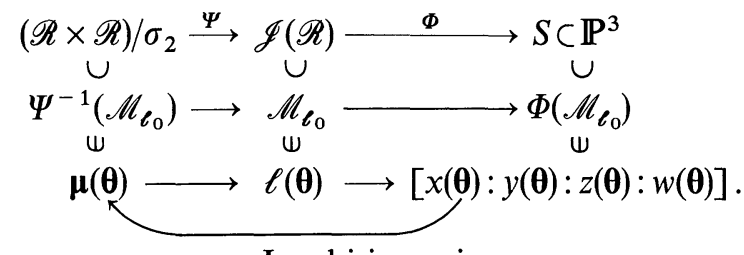

Jacobi inversion

We will now show that $\Phi\left(\mathscr{M}_{\ell_{0}}\right)$ is a two-dimensional real algebraic subvariety of $\mathbb{P}_{\mathbb{R}}^{3}$. In fact, $\Phi(\mathscr{M})$ coincides with the real points of the Kummer surface $S$. These results will be used in the next section to explicitly determine the real $\boldsymbol{\mu}$-locus. Part A below gives the topological description of $\Phi\left(\mathscr{M}_{\ell_{0}}\right)$; in Part B we discuss the real algebraic geometry of $\Phi\left(\mathscr{M}_{\ell_{0}}\right)$.

\section{A. Topology of $\Phi\left(\mathscr{M}_{\ell_{0}}\right)$}

We exploit the fact that $\Phi\left(\mathscr{M}_{\ell_{0}}\right)$ is the $2: 1$ image of the real two-torus $\mathscr{M}_{\ell_{0}}$; precisely, $\Phi(\mathbf{z})=\Phi(-\mathbf{z})$. Before proceeding, we remark that it suffices to determine the topology of $\Phi\left(\mathscr{M}_{\ell_{0}+\Delta}\right)$, where $\mathscr{M}_{\ell_{0}+\Delta}$ is the translate by $\Delta$ of $\mathscr{M}_{\ell_{0}}$ in $\mathscr{J}$. This is because translation by $\Delta$, a half period, jist induces a rigid motion of $\Phi\left(\mathscr{M}_{\ell_{0}}\right)$ in $\mathbb{P}^{3}$, which cannot alter the topology.

We recall from (II.D) the three cases of real $\Sigma_{2, j}^{(s)}$ and the associated list of all connected components $\mathscr{M}_{\ell_{0}}$, indexed by $\ell_{0}$, (II.12b). 
Kink-Kink Trains.

$$
\Sigma_{2,2}^{(s)}=\left\{E_{4}<E_{3}<E_{2}<E_{1}\right\}, \quad \ell_{0}= \pm\left(\frac{1}{4}, \frac{1}{4}\right), \pm\left(\frac{1}{4},-\frac{1}{4}\right) .
$$

Kink-Radiation Trains.

$$
\Sigma_{2,1}^{(s)}=\left\{E_{4}<E_{3}<0, E_{2}^{*}=E_{1}\right\}, \quad \ell_{0}= \pm\left(0, \frac{1}{4}\right) .
$$

Breather Trains.

$$
\Sigma_{2,0}^{(s)}=\left\{E_{4}^{*}=E_{3}, E_{2}^{*}=E_{1}\right\}, \quad \ell_{0}=(0,0) .
$$

Since the Kummer map $\Phi$ identifies $\ell+\Delta$ with $-(\ell+\Delta)$, and $\Delta$ is a half-period, the corresponding components $\mathscr{M}_{\ell_{0}+\Delta}, \mathscr{M}_{-\ell_{0}-\Delta}$ (or $\mathscr{M}_{\ell_{0}}, \mathscr{M}_{-\ell_{0}}$ ) are identified in $S$ :

$$
\Phi\left(\mathscr{M}_{\ell_{0}}\right)=\Phi\left(\mathscr{M}_{-\ell_{0}}\right) \text {. }
$$

Now, with the $\ell_{0}$ equivalence relation " " of Sect. II.D, it follows

$$
\begin{gathered}
\Phi: \mathscr{M}_{\ell_{0}} \stackrel{1: 1}{\longrightarrow} \Phi\left(\mathscr{M}_{\ell_{0}}\right) \text { if } \ell_{0} \approx \mathbf{0}, \\
\Phi: \mathscr{M}_{\ell_{0}} \stackrel{2: 1}{\longrightarrow} \Phi\left(\mathscr{M}_{\ell_{0}}\right) \text { if } \ell_{0} \sim \mathbf{0} .
\end{gathered}
$$

From these properties (IV.2a, b), and the fact (Proposition II.1) that each $\mathscr{M}_{\ell_{0}}$ is a real 2-torus, we have

Prooposition IV.1. The topological structure of the Kummer image, $\Phi(\mathscr{M})$, of the real isospectral class $\mathscr{M}$ for $\Sigma_{2, j}^{(s)}$, (IV.1), is

Kink-Kink Trains. $\Sigma_{2,2}^{(s)}: \Phi(\mathscr{M})$ is the union of two disjoint real 2-tori

$$
\Phi(\mathscr{M})=\Phi\left(\mathscr{M}_{(1 / 4,1 / 4)}\right) \cup \Phi\left(\mathscr{M}_{(-1 / 4,1 / 4)}\right) .
$$

Kink-Radiation Trains. $\Sigma_{2,1}^{(s)}: \Phi(\mathscr{M})$ is one real two-torus,

$$
\Phi(\mathscr{M})=\Phi\left(\mathscr{M}_{(0,1 / 4)}\right)=\Phi\left(\mathscr{M}_{(0,-1 / 4)}\right) .
$$

Breather Trains. $\Sigma_{2,0}^{(s)}: \Phi(\mathscr{M})$ is a "folded torus", i.e.

$$
\Phi(\mathscr{M})=\Phi\left(\mathscr{M}_{\mathbf{0}}\right)
$$

is homeomorphic to the quotient of the 2-torus, $\left(\theta_{1}, \theta_{2}\right) \in[0,2 \pi) \times[0,2 \pi)$, by the involution $\boldsymbol{\theta} \rightarrow-\boldsymbol{\theta}$. A fundamental domain of this quotient in $[0,2 \pi) \times[0,2 \pi)$ is depicted in Fig. 4.

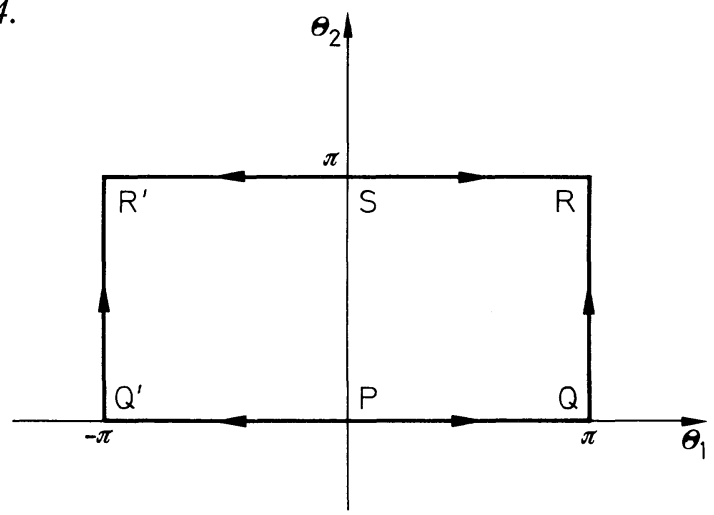

Fig. 4. Fundamental domain, breather Kummer surface 
$\Phi\left(\mathscr{M}_{\mathbf{0}}\right)$ is therefore homeomorphic to this fundamental domain with the edges identified by

$$
P Q \sim P Q^{\prime}, \quad Q R \sim Q^{\prime} R^{\prime}, \quad S R \sim S R^{\prime} .
$$

The points $P, S, Q=Q^{\prime}, R=R^{\prime}$ correspond to four nodes of the Kummer surface. A qualitative picture of $\Phi\left(\mathscr{M}_{\mathbf{0}}\right)$ is given by Fig. 5 .

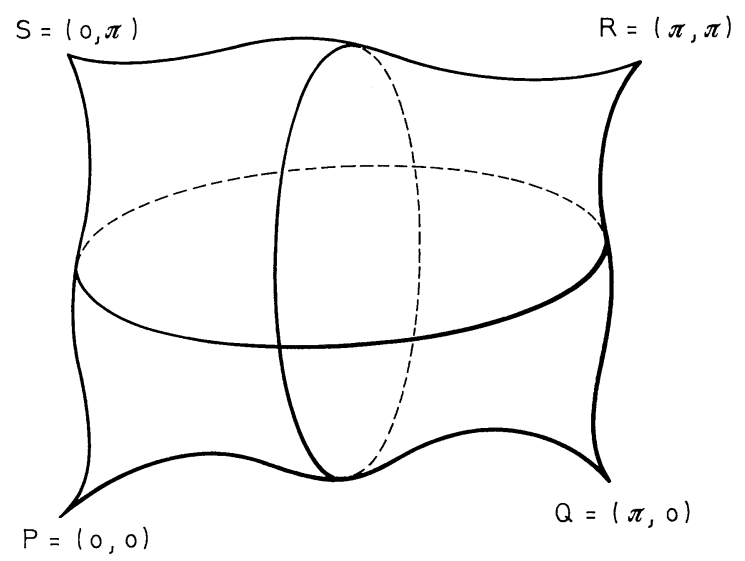

Fig. 5. Kummer surface image of the real breather torus

\section{B. The Real Kummer Surface Equations}

We now show that the image $\Phi\left(\mathscr{M}_{\ell_{0}}\right)$ has the structure of a real algebraic variety. That is, the Kummer surface equation (III.2), when restricted to a real component $\mathscr{M}_{\ell_{0}}$, is a real-valued equation on $\mathbb{P}_{\mathbb{R}}^{3}$.

For each $\mathscr{M}_{\ell_{0}}$ associated to $\Sigma_{2, j}^{(s)}$ in (IV.1), we must analyze the elements of (III.2): the quadratic theta function coordinates $[x: y: z: w]$,(III.1); the theta nulls $\alpha, \beta, \gamma, \delta$,(III.3); and the Kummer equation coefficients $A, B, C, D$,(III.4). To do so, we recall relevant information from Sect. II.B. Specifically: from (II.3), for all $\Sigma_{2, j}^{(s)}$,

$$
\operatorname{Re}\left(\ell(x, t)-\ell_{0}\right)=\mathbf{0} ;
$$

from (II.2), the period matrix $B$ for $\Sigma_{s, j}^{(s)}$ is given by

$$
\begin{aligned}
& \sum_{2,2}^{(s)}: \mathrm{B}=\left(\begin{array}{ll}
i b & i c \\
i c & i d
\end{array}\right), \\
& \sum_{2,1}^{(s)}: B=\left(\begin{array}{cc}
\frac{1}{2}+i b & i c \\
i c & i d
\end{array}\right), \\
& \sum_{2,0}^{(s)}: B=\left(\begin{array}{cc}
\frac{1}{2}+i b & i c \\
i c & \frac{1}{2}+i d
\end{array}\right), \\
& b, c, d \in \mathbb{R} .
\end{aligned}
$$

$\Phi\left(\mathscr{M}_{\ell_{0}}\right)$ is parametrized by the projective coordinates $[x: y: z: w]$ restricted to the locus

$$
\mathbf{z}=\ell(x, t)+\Delta=\left(\ell-\ell_{0}\right)+\left(\ell_{0}+\Delta\right) .
$$


Relations (IV.1), (IV.4) explicitly give the structure of (IV.6) for each real component.

We now have all the information necessary to analyze the elements of the Kummer equation restricted to real components. To begin with, the theta nulls $(\alpha, \beta, \gamma, \delta)=(x(\mathbf{0}), y(\mathbf{0}), z(\mathbf{0}), w(\mathbf{0}))$ are functions only of the period matrix $B$ for $\Sigma_{2, j}^{(s)}$. Using (IV.5), it is easy to calculate the properties of these parameters. For example, take $\Sigma_{2,2}^{(s)}, B$ given by (IV.5a), and compute

$$
\alpha=x(\mathbf{0})=\sum_{\mathbf{m} \in \mathbb{Z}^{2}} \exp [2 \pi i\{\langle B \mathbf{m}, \mathbf{m}\rangle\}]=\sum_{\mathbf{m} \in \mathbb{Z}^{2}} \exp \left[-2 \pi\left\{b m_{1}^{2}+2 c m_{1} m_{2}+d m_{2}^{2}\right\}\right],
$$

which is clearly real and positive. Proceeding this way for each case we determine:

$$
\begin{aligned}
& \Sigma_{2,2}^{(s)}: \alpha, \beta, \gamma, \delta \in \mathbb{R}^{+}, \\
& \Sigma_{2,1}^{(s)}: \alpha, \beta \in \mathbb{R} ; \quad \gamma, \delta \in e^{i \pi / 4} \mathbb{R}^{+}, \\
& \Sigma_{2,0}^{(s)}: \alpha \in \mathbb{R} ; \quad \beta, \gamma \in e^{i \pi / 4} \mathbb{R} ; \quad \delta \in \mathbb{R}^{+} .
\end{aligned}
$$

The Kummer coefficients, by (III.4), are then easily analyzed from these conditions on the theta nulls. Let $\widetilde{A}, \tilde{B}, \widetilde{C}, \tilde{D} \in \mathbb{R}$, then

$$
\begin{array}{llll}
\Sigma_{2,2}^{(s)}: A=\tilde{A}, & B=\tilde{B}, & C=\tilde{C}, & D=\tilde{D}, \\
\Sigma_{2,1}^{(s)}: A=i \tilde{A}, & B=i \tilde{B}, & C=\tilde{C}, & D=i \tilde{D}, \\
\Sigma_{2,0}^{(s)}: A=\tilde{A}, & B=i \tilde{B}, & C=i \tilde{C}, & D=\tilde{D} .
\end{array}
$$

The coordinates $x, y, z, w$ are analyzed in the same manner using the information summarized above. For example, for $\Sigma_{2,2}^{(s)}$, by (IV.6),

$$
\mathbf{z}=\frac{1}{2 \pi}\left(\theta_{1} \mathbf{f}_{1}+\theta_{2} \mathbf{f}_{2}\right)+\left(\frac{ \pm e_{1} \pm e_{2}}{4}\right),
$$

and the elements of $B$ and $\mathbf{f}_{i}$ are purely imaginary, so

$$
\begin{aligned}
y(\mathbf{z})= & \sum_{\mathbf{m} \in \mathbb{Z}^{2}} \exp \left[4 \pi i\left\langle\mathbf{m}+\frac{e_{1}}{2}, \frac{ \pm e_{1} \pm e_{2}}{4}\right\rangle\right] \\
& \cdot \exp \left[2 \pi i\left\{\langle B \mathbf{m}, \mathbf{m}\rangle+2\left\langle\mathbf{m}, \frac{1}{2 \pi}\left(\theta_{1} \mathbf{f}_{1}+\theta_{2} \mathbf{f}_{2}\right)\right\rangle\right\}\right] \\
= & \pm i \sum_{\mathbf{m} \in \mathbb{Z}^{2}}(-1)^{m_{1}+m_{2}} \exp \left[2 \pi i\left\{\langle B \mathbf{m}, \mathbf{m}\rangle+2\left\langle\mathbf{m}, \frac{1}{2 \pi}\left(\theta_{1} \mathbf{f}_{1}+\theta_{2} \mathbf{f}_{2}\right)\right\rangle\right\}\right] \\
& \in i \mathbb{R} .
\end{aligned}
$$

The result is: with $X, Y, Z, W \in \mathbb{R}$,

$$
\begin{array}{ll}
\Sigma_{2,2}^{(s)}: x=X, \quad y=i Y, \quad z=i Z, \quad w=W, \\
\Sigma_{2,1}^{(s)}: x=e^{i \pi / 4} X, \quad y=e^{i \pi / 4} Y, \quad z=i Z, \quad w=W, \\
\Sigma_{2,0}^{(s)}: x=i X, \quad y=e^{i \pi / 4} Y, \quad z=e^{i \pi / 4} Z, \quad w=W .
\end{array}
$$

We can now evaluate the Kummer surface equation for each real isospectral class $\Sigma_{2, j}^{(s)}$. With real coordinates $X, Y, Z, W$ and real coefficients $\widetilde{A}, \widetilde{B}, \widetilde{C}, \widetilde{D}$, we have the following 
Proposition IV.2. The Kummer surface equation, (III.2), restricted to the image $\Phi(\mathscr{M})$ of the real isospectral class $\mathscr{M}$ for each $\Sigma_{2, j}^{(s)}$, becomes:

$\Sigma_{2,2}^{(s)}$ : Kink-Kink Trains,

$$
\begin{aligned}
& X^{4}+Y^{4}+Z^{4}+W^{4}+\tilde{A}\left(X^{2} W^{2}+Y^{2} Z^{2}\right)-\tilde{B}\left(Y^{2} W^{2}+X^{2} Z^{2}\right) \\
& -\tilde{C}^{2}\left(Z^{2} W^{2}+X^{2} Y^{2}\right)-2 \tilde{D} X Y Z W=0 .
\end{aligned}
$$

The zero locus consists entirely of the two disjoint topological tori in (IV.3a).

$\Sigma_{2,1}^{(s)}$ : Kink-Radiation Trains

$$
\begin{gathered}
-X^{4}-Y^{4}+Z^{4}+W^{4}+\tilde{A}\left(X^{2} W^{2}+Y^{2} Z^{2}\right)-\tilde{B}\left(Y^{2} W^{2}+X^{2} Z^{2}\right) \\
-\tilde{C}\left(Z^{2} W^{2}-X^{2} Y^{2}\right)-\tilde{D} X Y Z W=0 .
\end{gathered}
$$

The zero locus consists of just the topological torus in (IV.3b).

$\Sigma_{2,0}^{(s)}:$ Breather Trains,

$$
\begin{gathered}
X^{4}-Y^{4}-Z^{4}+W^{4}-\tilde{A}\left(X^{2} W^{2}+Y^{2} Z^{2}\right)-\tilde{B}\left(Y^{2} W^{2}-X^{2} Z^{2}\right) \\
-\tilde{C}\left(Z^{2} W^{2}-X^{2} Y^{2}\right)-2 \tilde{D} X Y Z W=0 .
\end{gathered}
$$

The zero locus is a two-sheeted surface branched at the four nodes it contains, as explicitly shown in Fig. 5. In this case, $\Phi(\mathscr{M})$ is one of the sheets of this surface.

We close this section with an observation about the branch point representation, in terms of fundamental quadrics, as given by (III.20). Using the values of the theta nulls in (IV.7), we can determine the value of $k$ in (III.20) which makes the branch points $E_{1}, E_{2}, E_{3}, E_{4}$ coincide with $\Sigma_{2, j}^{(s)}$ as described in Sect. II.B. The result is

$$
\Sigma_{2,2}^{(s)}: k=1, \quad \sum_{2,1}^{(s)}: k=e^{i \pi / 4}, \quad \sum_{2,0}^{(s)}: k=i
$$

\section{Parametrization of the $\mu$ Trajectories}

In this section we explicitly compute the $\boldsymbol{\mu}$-loci for real flows by restricting the Jacobi inversion formulae (III.13a, b) to the real Kummer surface. We illustrate this for specific choices of $\Sigma_{2, j}^{(s)}, j=0,1,2$, which correspond to special Kummer surfaces called tetrahedroids. The name derives from classical geometry because the nodes, in addition to the usual 16:6 configuration (III.E), are also partitioned into four sets of coplanar nodes. The four planes so distinguished form a tetrahedron.

For a tetrahedroid, one can always perform a linear change of coordinates so that $D=0$ [22]. We do this in each case below. The resulting Kummer equation, (III.4), then involves only the squares of $x, y, z, w$. This equation arises in optics as the dispersion relation for double refraction of electromagnetic waves in a biaxial crystal $[24,25]$ ( $w$ is the frequency and $x, y, z$ are wave numbers). In this context, the tetrahedroid is referred to as a wave surface.

The additional structure of tetrahedroids is due to special properties of the curve $\mathscr{R}$ and the Jacobian $\mathscr{J}(\mathscr{R})$. Specifically: the branch points of $\mathscr{R}$ are invariant under holomorphic involution iff the hyperelliptic (genus 2) Jacobian factors into 
the product of elliptic (genus 1) Jacobians. This is the geometric framework of "separable solutions" $[4,22]$ in the soliton literature. These are two-phase (genus 2) solutions expressible in terms of elliptic functions on two genus 1 curves. In [4], Forest and McLaughlin explicitly showed that, when $\Sigma_{2, j}^{(s)}$ is invariant under the involution $E \rightarrow 1 / 16^{2} E$, the Jacobian factors into the product of elliptic Jacobians. Moreover, they show that such involutions characterize boundary conditions of the potentials.

We now present explicit examples of how the developments of this paper yield an explicit computation of the $\boldsymbol{\mu}$ trajectories for the flows of real potentials. The surfaces we choose are in fact double tetrahedroids [22] (tetrahedroids in two distinct ways), corresponding to highly symmetric $\Sigma_{2, j}^{(s)}$ with one real degree of freedom. We list the necessary data for each case, and then the plots of the $\boldsymbol{\mu}$-loci generated by the parametrization on the real wave surfaces. The reader should refer to the real Kummer data in Sect. IV. (Details of these calculations and others like them are available upon request.)

Kink-Kink Trains.

$\Sigma_{2,2}^{(s)}: E_{1}=-1, \quad E_{2}=-\left(1+2 / \hat{\alpha}^{2}\right) \quad E_{3}=-\hat{\alpha}^{2} /\left(\hat{\alpha}^{2}-2\right), \quad E_{4}=-\left(\hat{\alpha}^{2}+2\right) /\left(\hat{\alpha}^{2}-2\right)$.

Coordinates.

$$
\begin{gathered}
\hat{x}=x+w=\hat{X}, \quad \hat{y}=y+z=i \hat{Y}, \quad \hat{z}=y-z=i \hat{Z}, \quad \hat{w}=x-w=\hat{W}, \\
\text { where } \hat{X}, \hat{Y}, \hat{Z}, \hat{W} \in \mathbb{R} .
\end{gathered}
$$

Theta Nulls.

$$
\hat{\alpha}=\alpha+\delta, \quad \hat{\beta}=\beta+\gamma, \quad \hat{\gamma}=\beta-\gamma, \quad \hat{\delta}=\alpha-\delta .
$$

Tetrahedroid Constraints.

$$
\hat{\alpha}>\sqrt{2}, \quad \hat{\beta}=\hat{\delta}=1, \quad \hat{\gamma}=0 .
$$

Coefficients.

$$
\hat{A}=-\hat{\alpha}^{2}, \quad \hat{B}=\hat{\alpha}^{4}-2, \quad \hat{C}=-\hat{\alpha}^{2}, \quad \hat{D}=0 .
$$

Real Kummer Equation.

$$
\begin{aligned}
F= & \hat{X}^{4}+\hat{Y}^{4}+\hat{Z}^{4}+\hat{W}^{4}-\hat{\alpha}^{2}\left(\hat{X}^{2}-\hat{Z}^{2}\right)\left(\hat{W}^{2}-\hat{Y}^{2}\right) \\
& +\left(2-\hat{\alpha}^{4}\right)\left(\hat{X}^{2} \hat{Z}^{2}+\hat{Y}^{2} \hat{W}^{2}\right)=0 .
\end{aligned}
$$

Inversion Formulae of $\boldsymbol{\mu}$ Constrained to $F=0$.

$$
\mu_{1} \mu_{2}=\left(\frac{\hat{\alpha}^{2}+2}{\hat{\alpha}^{2}-2}\right) \frac{(\hat{\alpha} \hat{W}-\hat{X})+i \hat{Z}}{(\hat{\alpha} \hat{W}-\hat{X})-i \hat{Z}}
$$

$\mu_{1}+\mu_{2}$

$$
=\frac{\frac{-2}{\left(\hat{\alpha}^{2}-2\right)}\left\{\left(\hat{\alpha}^{2}-1\right)[\hat{\alpha} \hat{W}-\hat{X}+i \hat{Z}]+\hat{\alpha}[\hat{W}-i(\hat{Y}+\hat{\alpha} \hat{Z})]-\left(1-2 / \hat{\alpha}^{2}\right)[\hat{X}+i(\hat{Z}-\hat{\alpha} \hat{Y})]\right\}}{(\hat{\alpha} \hat{W}-\hat{X})-i \hat{Z}} .
$$




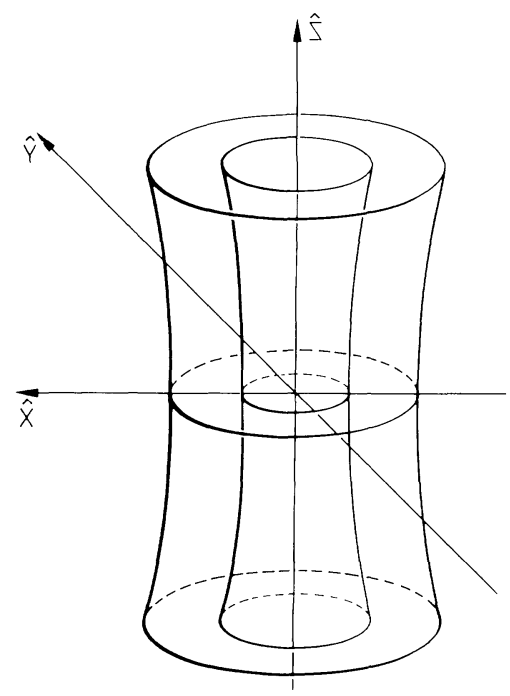

Fig. 6a. Kummer image: real kink-kink isospectral manifold (in affine space $\hat{W} \neq 0$ )

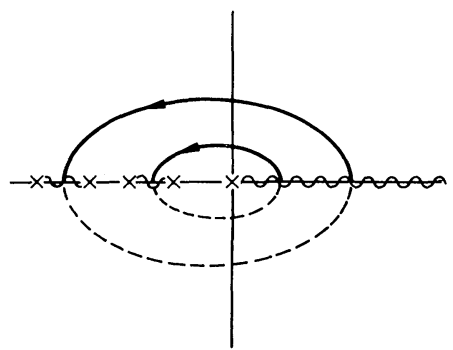

Fig. 6b

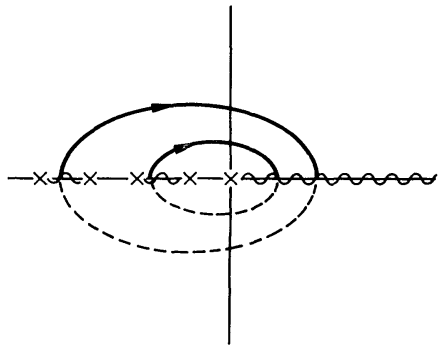

$\vec{\mu}$-loci inner torus

\section{Kink-Radiation Trains.}

$$
\Sigma_{2,1}^{(s)}: E_{1}=-\frac{\alpha^{2}}{2}, \quad E_{2}=-\left(\frac{\alpha^{2}}{2}+\frac{2}{\alpha^{2}}\right), \quad E_{3}=-\frac{\alpha^{2}}{2}+i, \quad E_{4}=-\frac{\alpha^{2}}{2}-i .
$$

Coordinates.

$$
x=e^{-i \pi / 4} X, \quad y=e^{i \pi / 4} Y, \quad z=i Z, \quad w=W, \quad X, Y, Z, W \in \mathbb{R} .
$$

Tetrahedroid Constraints.

$$
\alpha \in \mathbb{R}-\{0\}, \quad \beta=0, \quad \gamma=\delta=e^{i \pi / 4} .
$$

\section{Coefficients.}

$$
A=i \alpha^{2}, \quad B=i \alpha^{2}, \quad C=-\left(\alpha^{4}+2\right), \quad D=0 .
$$


Real Kummer Equation.

$$
\begin{aligned}
F= & -X^{4}-Y^{4}+Z^{4}+W^{4}+\alpha^{2}\left(-X^{2}+Y^{2}\right)\left(Z^{2}-W^{2}\right) \\
& +\left(\alpha^{4}-2\right)\left(-X^{2} Y^{2}+Z^{2} W^{2}\right)=0 .
\end{aligned}
$$

Inversion Formulae of $\boldsymbol{\mu}$ Constrained to $F=0$.

$$
\begin{aligned}
\mu_{1} \mu_{2}=\left(\frac{\alpha^{4}}{4}+1\right) \frac{(\alpha W-X)-i Y}{(\alpha W-X)+i Y}, \\
\mu_{1}+\mu_{2}=\frac{\left\{-\left(\alpha^{4}+2+i \alpha^{2}\right)(\alpha W-X-i Y)+\left(i \alpha^{2}-2\right)\right.}{\left(\alpha^{2}+2 i\right)} \\
\\
\frac{\left.\cdot(X-i(Y+\alpha Z))-2 i \alpha\left(\frac{\alpha^{2}}{2}+\frac{2}{\alpha^{2}}\right)(\alpha Y+W-i Z)\right\}}{\cdot(\alpha W-X+i Y)} .
\end{aligned}
$$

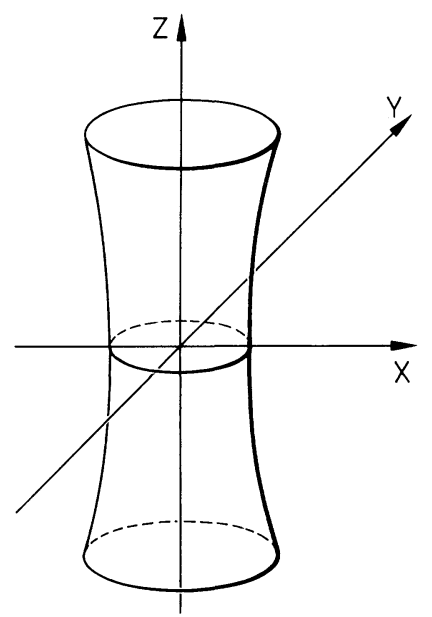

Fig. 7a. Kummer image: real kink-radiation manifold
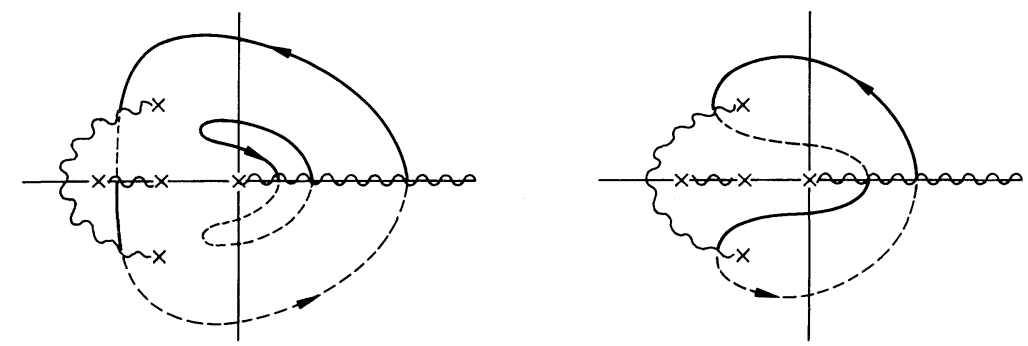

Fig. 7 b. $\mu$-loci for two independent cycles on $m$

Breather Trains.

$$
\Sigma_{2,0}^{(s)}: E_{1}=\frac{4 i\left(1-\alpha^{2}\right)}{\alpha^{2}}, \quad E_{2}=\frac{4 i\left(1+\alpha^{2}\right)}{\alpha^{2}}, \quad E_{3}=E_{2}^{*}, \quad E_{4}=E_{1}^{*} .
$$


Coordinates.

$$
\begin{gathered}
\tilde{x}=x+w=i X+W, \quad \tilde{y}=y+z=e^{i \pi / 4}(Y+Z), \\
\tilde{z}=y-z=e^{i \pi / 4}(Y-Z), \quad \tilde{w}=x-w=i X-W, \\
X, Y, Z, W \in \mathbb{R}, \quad X>0 .
\end{gathered}
$$

Theta Nulls.

$$
\hat{\alpha}=\alpha+\delta, \quad \hat{\beta}=\beta+\gamma, \quad \hat{\gamma}=\beta-\gamma, \quad \hat{\delta}=\alpha-\delta .
$$

Tetrahedroid Constraints.

$$
\tilde{\alpha}=\sqrt{2} e^{i \pi / 4} \hat{\alpha}, \quad \tilde{\beta}=2 e^{i \pi / 4}, \quad \tilde{\gamma}=0, \quad \tilde{\delta}=\sqrt{2} e^{-i \pi / 4} \hat{\alpha} .
$$

Coefficients.

$$
\tilde{A}=2\left(1-\frac{2}{\tilde{\alpha}^{2}}\right), \quad \tilde{B}=\frac{2}{\tilde{\alpha}^{2}}, \quad \tilde{C}=-\tilde{B}, \quad \tilde{D}=0 .
$$

Real Kummer Equation.

$$
\begin{aligned}
F= & \left(X^{2}-W^{2}\right)^{2}-\left(Y^{2}+Z^{2}\right)^{2}-4\left(W^{2} X^{2}+Y^{2} Z^{2}\right)+\left(1-\frac{2}{\tilde{\alpha}^{4}}\right) \\
& \cdot\left[\left(X^{2}+W^{2}\right)^{2}-\left(Y^{2}-Z^{2}\right)^{2}\right]+\frac{16}{\tilde{\alpha}^{2}} X Y Z W=0 .
\end{aligned}
$$

Inversion Formulae of $\boldsymbol{\mu}$ Constrained to $F=0$.

$$
\begin{aligned}
\mu_{1} \mu_{2}=\frac{16\left(1-\tilde{\alpha}^{4}\right)}{\tilde{\alpha}^{4}}\left\{\frac{\tilde{\alpha}(X-W)+i(Y-Z)}{\tilde{\alpha}(X-W)-i(Y-Z)}\right\}, \\
\mu_{1}+\mu_{2}=\frac{-8 i[\tilde{\alpha}(X-W)+i(Y-Z)]+2 \sqrt{2} i\left(\tilde{\alpha}^{-1}-\tilde{\alpha}\right)}{\tilde{\alpha}(X-W)} \\
\\
\left.-i \sqrt{2} \tilde{\alpha}\{Y+Z-i(Y-Z)\}-2 e^{i \pi / 4}(i X-W)\right] \\
+\frac{4\left(\tilde{\alpha}^{-1}+\tilde{\alpha}\right)\left[-2 e^{i \pi / 4}(i X+W)-\sqrt{2} \tilde{\alpha}[(Y-Z)-i(Y+Z)]\right]}{\tilde{\alpha}(X-W)-i(Y-Z)} .
\end{aligned}
$$

The breather wave surface is one sheet $(X>0)$ of the folded torus, Fig. 5.

We choose this model case to provide several computer plots which illustrate the range of real $\boldsymbol{\mu}$-loci for breather trains. The following plots represent selected cycles from the fundamental domain, Fig. 4. Branch cuts are not drawn in, but follow the convention of Fig. 2. Plot 1 is the $\mu_{1}$-locus for a cycle $\theta_{2} \equiv$ constant. Plot 2 is the $\mu_{2}$-locus for $\theta_{1} \equiv$ const. (The other $\mu_{j}$ locus, in each case, is contractible to a point.) These first two plots are therefore a basis for the real $\boldsymbol{\mu}$ homology, $a_{1}-2 b_{1}$ and $a_{2}-2 b_{2}$. Plots 3,4 show the $\mu_{1}$ and $\mu_{2}$ paths for a choice $\theta_{2} \equiv$ const, $\theta_{1} \equiv$ const, respectively, where one can observe the contractible cycle. Plot 5 corresponds to a flow along one diagonal of the fundamental domain. Plot 6 consists of $\mu_{1}$ and $\mu_{2}$ loci for six distinct, but homologically equivalent, flows, $\theta_{2} \equiv c_{i}, i=1, \ldots, 6$, Plot 7 

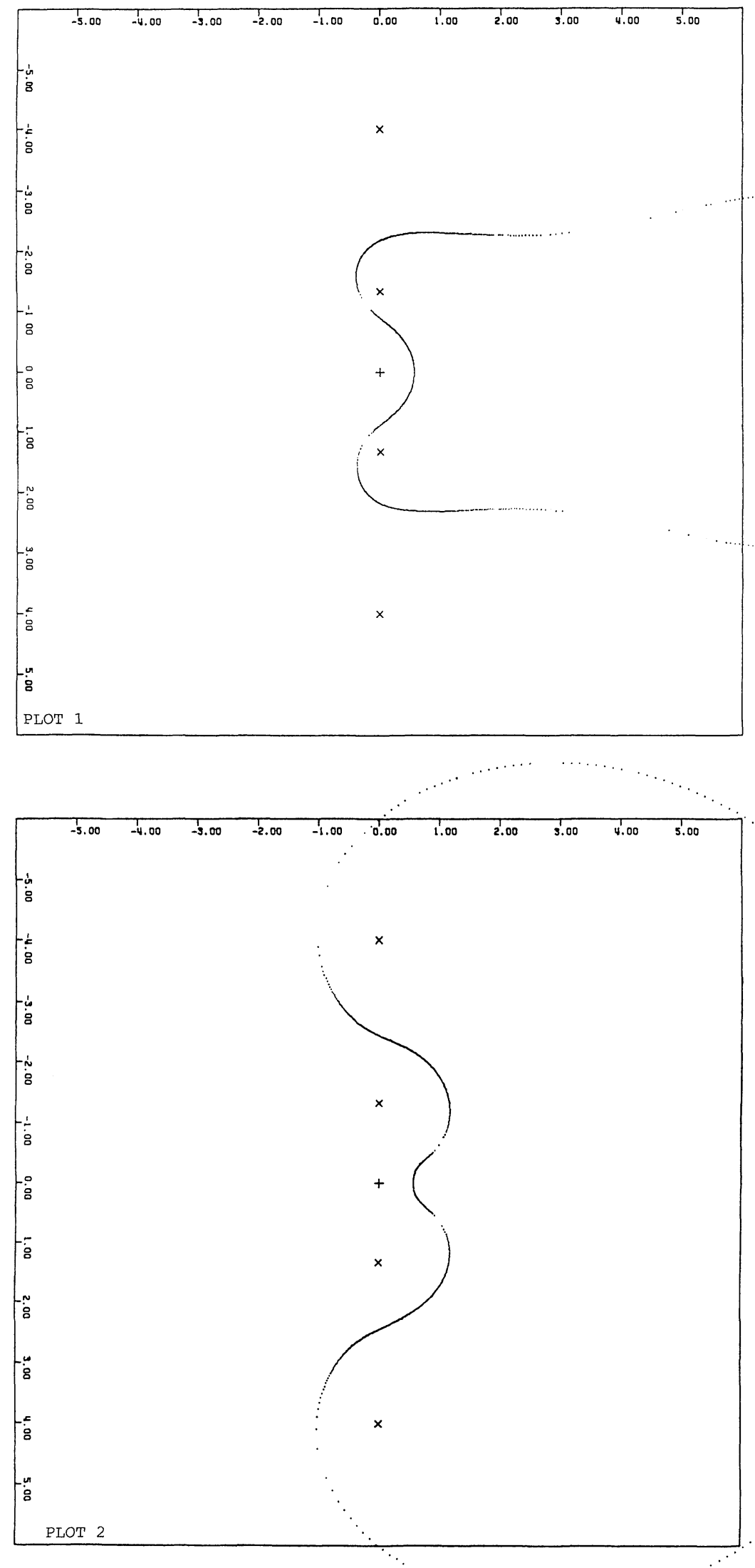

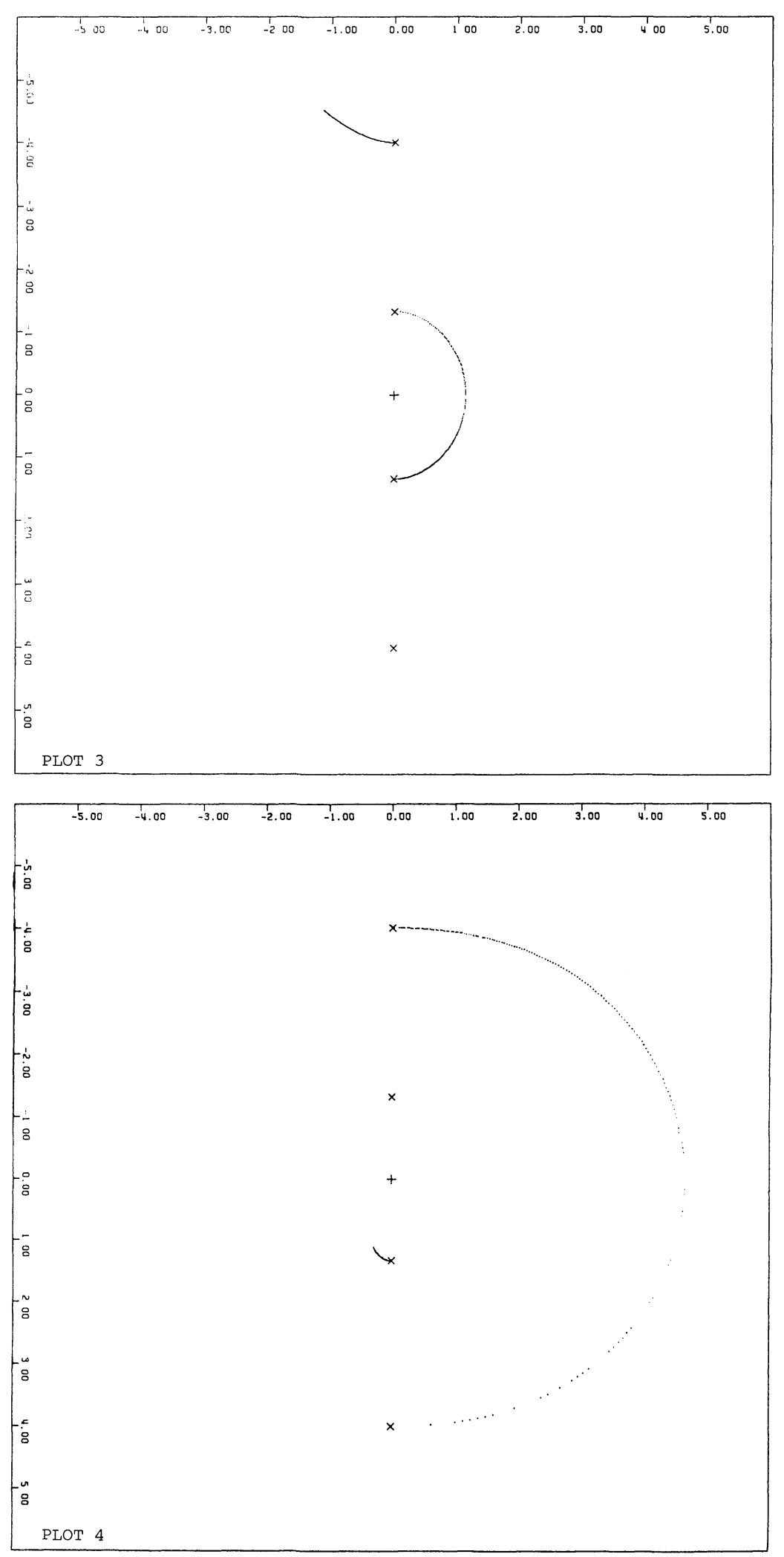

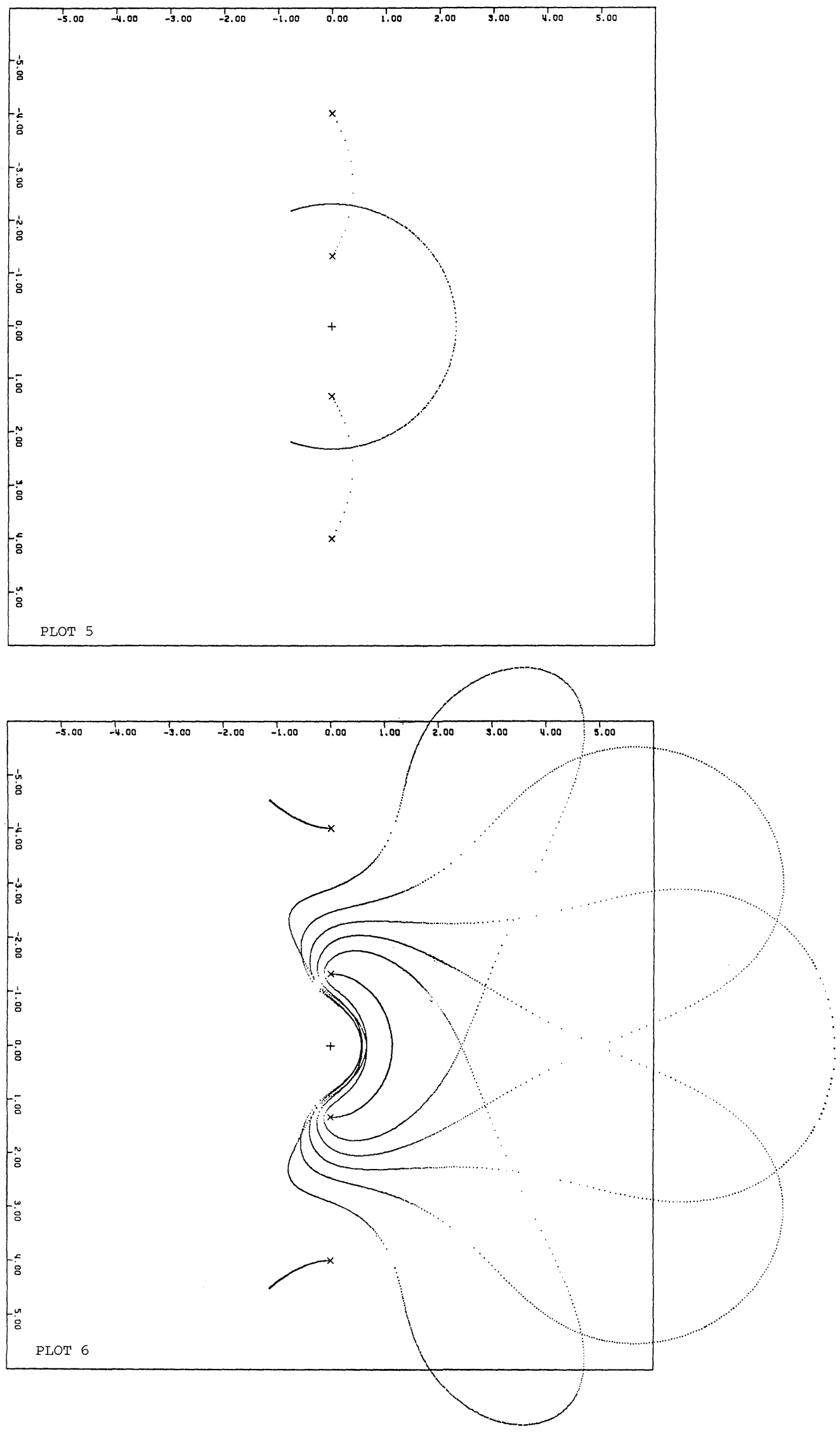


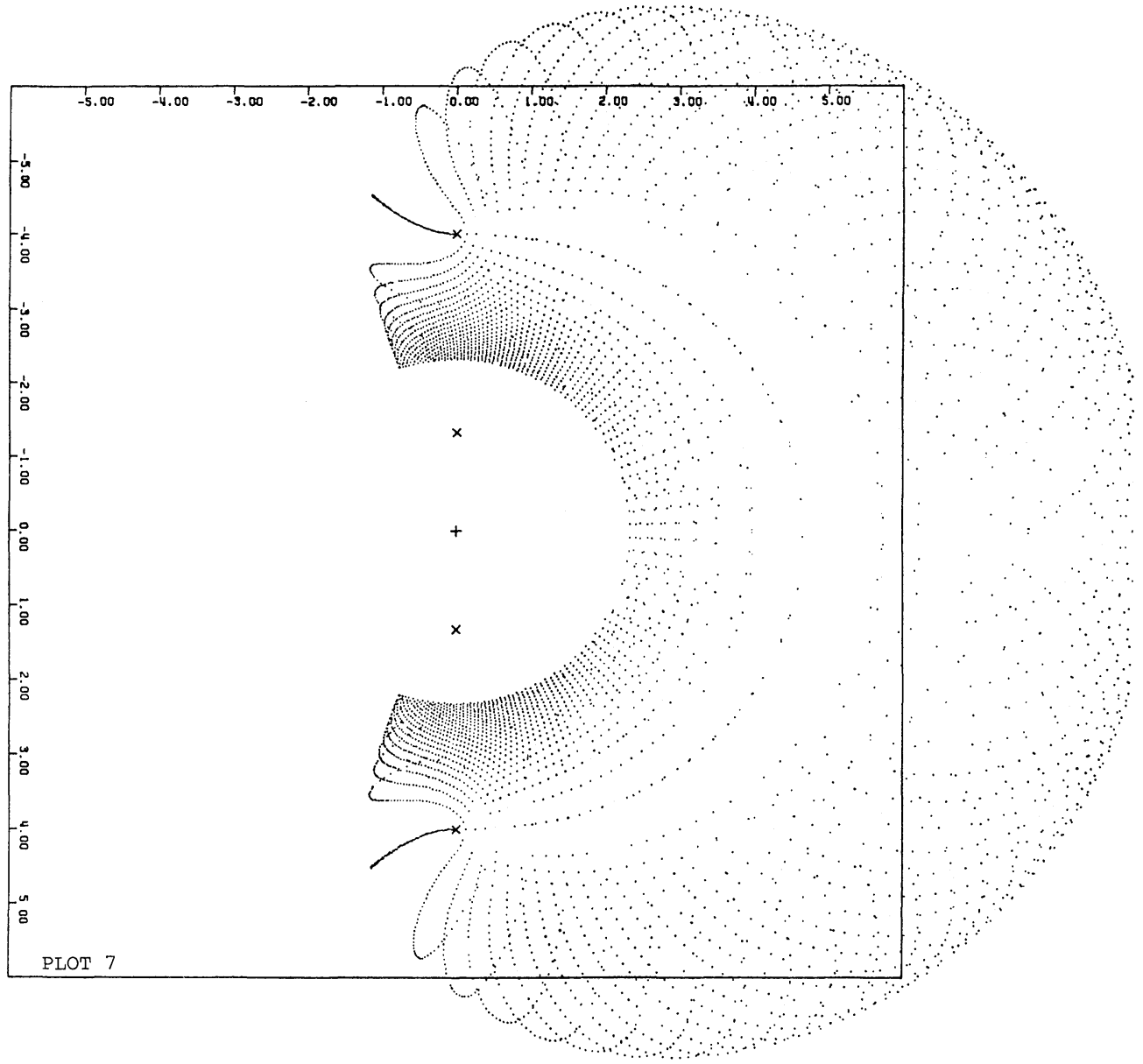

consists of the " $\mu_{1}$-blob", the projection of all $\mu_{1}$ values on $\mathbb{C}$ for a uniform sampling of points from the full fundamental domain. Plot 8 is the " $\mu$-blob", a portrait of all values assumed by $\mu_{1}$ and $\mu_{2}$ over the same grid as in Plot 7 .

\section{Concluding Remarks}

The real isospectral manifolds of $N$ phase quasiperiodic sine-Gordon potentials are characterized for $N<\infty$. Global coordinates for these manifolds are provided by the spectral $\boldsymbol{\mu}$ variables, defined via quadratic eigenfunctions, and the $\boldsymbol{\mu}$ dynamics is deduced.

The algebro-geometric methods presented here are shown as a natural setting to answer the questions addressed in Sect. I. It is hoped that these methods, in particular the role of the Kummer variety, will find a more familiar place in the 


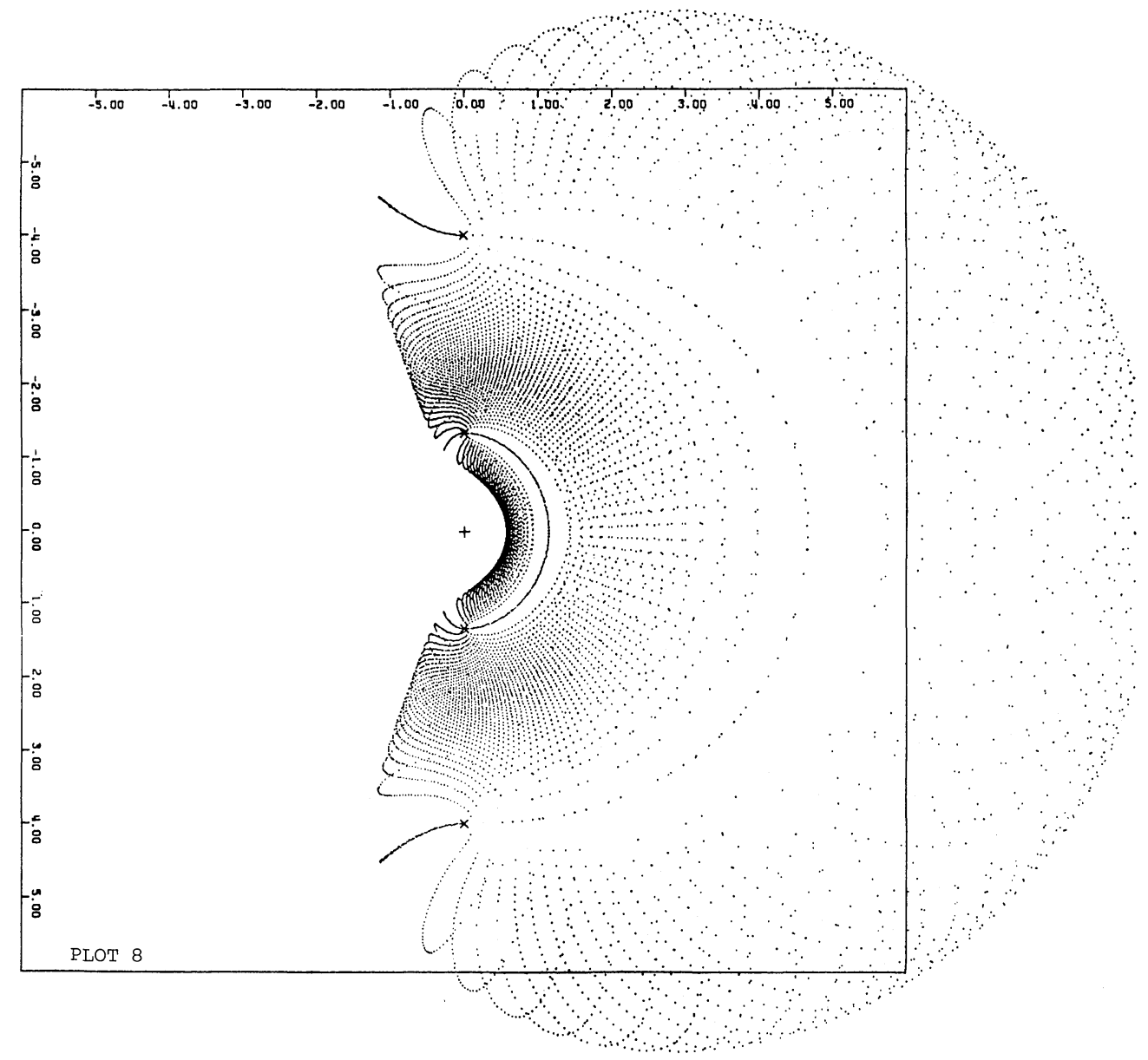

study of integrable systems and their perturbations. We close with a few remarks on the context of this paper in related research efforts.

Our interest in the global dynamical behavior of the sine-Gordon $\boldsymbol{\mu}$ variables originated in attempts to study modulations and perturbations of $N$ phase sineGordon wavetrains. In our previous work on $\mathrm{KdV}$ [27] and sinh-Gordon [6] modulation theory, the complete control of the $\mu$ 's under real flows was crucial. With the results of this paper, the sine-Gordon modulation theory is now complete [17].

In the context of perturbation theory, the $\mu$ variables together with the periodic spectrum $\left\{E_{i}\right\}$ provide canonical variables on a space $\mathscr{F}$ of potentials (functions in $x)$ which are periodic with fixed period [11]. These in turn provide a natural basis of $\mathscr{F}$ with which to investigate the linearized stability of the perturbed equations. Once again, to carry out this perturbation scheme, one needs to understand how reality constrains the $\boldsymbol{\mu}$-trajectories. Here one discovers a striking feature of sine- 
Gordon, not seen in $\mathrm{KdV}$, which is a direct consequence of the $\boldsymbol{\mu}$-geometry: it is possible to find solutions of linearized sine-Gordon, near an $N$ phase potential, which grow exponentially in time. This instability is a generalization of the classical Benjamin-Feir instability [18].

Of course, a complete treatment of perturbation theory for periodic sineGordon requires one to deal with infinite genus Riemann surfaces. Here, the reality question is more complicated. The Abel map is now given by an infinite sum whose convergence depends on controlling the size of the $\mu$ 's. Granted a well-defined Abel map, the analysis of reality presented here extends to $N=\infty$. We feel that the infinite genus Kummer variety, defined through squared eigenfunctions along the lines of Appendix B, can play a role in resolving these convergence problems.

There are other results which relate to the structure presented here. Previato [28] has characterized the real $N$ phase isospectral manifold for the focusing nonlinear Schrödinger (NLS) theory. Her method, following the work of Deift, Lund, and Trubowitz [29], associates a complex $N$-dimensional Neumann system to a given $N$ phase solution. The Neumann model is built out of the simple eigenfunctions of the AKNS system. A level set of the Neumann integrals is the Jacobian of the NLS curve $\mathscr{R}$. The real isospectral class then consists of the fixed points of an antiholomorphic involution induced by the real symmetries of $\mathscr{R}$.

\section{Appendix A. Kummer Coefficients, Theta Nulls, and Branch Points}

In this appendix we derive the coefficients $A, B, C, D$ of the Kummer surface equation as functions of the simple periodic spectra $E_{1}, E_{2}, E_{3}, E_{4}$. The derivation is for general genus 2 hyperelliptic curves.

We emphasize one point first. Coordinates have been fixed on $\mathscr{J}$, and therefore on $S$, in Sect. III. This choice of coordinates is determined by the homology basis (Figs. 3-5) and the base point in $\mathscr{J}$ (Table 1). Therefore, the branch points $E_{1}, E_{2}, 0, \infty, E_{3}, E_{4}$ are unambiguously represented in $S$.

Given this fact, it becomes clear that special symmetries and features of the Kummer surface only reveal themselves in the proper choice of coordinates. This is where a choice of homology basis (i.e., choice of "cut structure") becomes relevant. Said differently, only the tractability of information about theta functions and geometry of $S$ is affected by the choice of coordinates.

The derivation of $A, B, C, D$ in terms of the branch points of $\mathscr{R}$ is based on the Cayley irrational representations of $S$ (see Hudson [22]). We outline the construction of one Cayley representation of $S$. From the orthogonal matrix of tropes, Sect. III, the second and third columns are orthogonal,

$$
(a c)(b a)-(d a)(c c)+(c d)(d b)-(b b)(a d)=0 .
$$

Moreover, when evaluated at the node $[\alpha: \beta: \gamma: \delta]$, the corresponding quadratic relation is [see (III.7)]

$$
[d a][c c]-[c d][d b]+[b b][a d]=0 .
$$

Next consider three constants $\lambda, \mu, v$ such that

$$
\lambda+\mu+v=0,
$$


and the following four quartic surfaces:

$$
\begin{gathered}
\sqrt{-\lambda(d a)(c c)}+\sqrt{\mu(c d)(d b)}+\sqrt{-v(b b)(a d)}=0, \\
\sqrt{\lambda(a c)(b a)}+\sqrt{-\mu(b b)(a d)}+\sqrt{v(a d)(d b)}=0, \\
\sqrt{-\lambda(b b)(a d)}+\sqrt{\mu(a c)(b a)}+\sqrt{-v(d a)(c c)}=0, \\
\sqrt{\lambda(c d)(d b)}+\sqrt{-\mu(d a)(c c)}+\sqrt{v(a c)(b a)}=0 .
\end{gathered}
$$

In view of $(\mathrm{A} .1 \mathrm{a}, \mathrm{b}, \mathrm{c})$, it follows that each of these quartic surfaces has the eight planes

$$
(a c)=(b a)=(d a)=(c c)=(c d)=(b d)=(b b)=(a d)=0
$$

for tropes, and thus the eight common points for nodes. The projective space of $m$ degree surfaces in $\mathbb{P}^{n}$ has dimension $\left(\begin{array}{c}m+n \\ n\end{array}\right)-1$, so the projective space of quartic surfaces in $\mathbb{P}^{3}$ has $\operatorname{dim}=34$. Forcing the surface to pass through a node imposes 4 conditions, so that the eight nodes impose 32 conditions on this space, which are independent. By imposing one more node on this space, a unique quartic surface is determined, which is invariant under the automorphisms described in the text, and therefore is the Kummer surface. One can check that the node $[\alpha: \beta: \gamma: \delta]$ does not pass through the four quartics in (A.2) for arbitrary $\lambda+\mu+v=0$; imposing this node yields

$$
(\lambda: \mu: v)=(-[d a][c c]:[c d][d b]:-[b b][a d]) .
$$

The result is the:

Cayley Irrational Representation of $S$.

$$
\sqrt{[c d][d b](c d)(d b)}+\sqrt{[b b][a d](b b)(a d)}+\sqrt{[d a][c c](d a)(c c)}=0 .
$$

Consider the tangent cone to $S$ at the node $[\gamma: \delta:-\alpha:-\beta]$. Six tropes pass through this node, and each is tangent to this cone. By duality, the normals to these tropes are projectively equivalent to the branch points of $\mathscr{R}$ (Table 1 , Sect. III). Thus, in our notation,

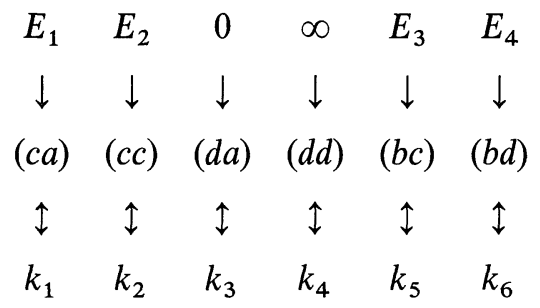

which serves as the definition of $k_{1}, \ldots, k_{6}$.

Next consider the Cayley representation of $S$ in (A.3). The elements $k_{1}, \ldots, k_{6}$ in (A.4) lie on the intersection of $S$ with the fundamental trope $(a b)=0$. We bring the trope $(a b)$ to the forefront by applying the automorphism (ac) to (A.3), which yields

$$
\sqrt{[c d][d b](b c)(a a)}+\sqrt{[b b][a d](c a)(d c)}+\sqrt{[d a][c c](a b)(b d)}=0 .
$$


The intersection of (A.5) with $(a b)=0$ is a "singular" conic (Sect. III), which is isomorphic to $\mathbb{P}^{1}$. This isomorphism can be realized by restricting any linear fractional function on $\mathbb{P}^{3}$ to the conic. In particular, we realize this map by any ratio of tropes which does not vanish on the singular conic. This map carries the nodes in (A.4) to six elements in $\mathbb{P}^{1}$, which therefore are projectively equivalent to the branch points. Different mappings will yield different sixtuples of elements in $\mathbb{P}^{1}$, but all are equivalent up to linear fractional transformations.

In particular, consider the map $\frac{(b c)}{(d c)}$ evaluated at the nodes in (A.4). At points where this function is apparently indeterminate, specifically along $(a b)=0$, (A.5) yields

$$
\frac{(b c)}{(d c)}=\frac{[b b][a d]}{[c d][d b]} \frac{(c a)}{(a a)} .
$$

From Sect. III,

$$
\begin{aligned}
& \frac{(b c)}{(d c)}=\frac{\gamma x+\delta y-\alpha z-\beta w}{\alpha x+\beta y-\gamma z-\delta w}, \\
& \frac{(c a)}{(a a)}=\frac{\beta x-\alpha y-\delta z+\gamma w}{\delta x-\gamma y-\beta z+\alpha w} .
\end{aligned}
$$

Referring to Table 1 and (III.7), we evaluate $(b c) /(d c)$ at the nodes to obtain

$$
\begin{array}{ll}
E_{1} \rightarrow \frac{[b b][a d][d d]}{[c d][d b][b d]}=k_{1}, & \infty \rightarrow 0=k_{4}, \\
E_{2} \rightarrow \frac{[a d]}{[c d]}=k_{2}, & E_{3} \rightarrow \frac{[d d]}{[b d]}=k_{5}, \\
0 \rightarrow \frac{[b b]}{[d b]}=k_{3}, & E_{4} \rightarrow \infty=k_{6} .
\end{array}
$$

The linear fractional transformation

$$
\omega(z)=k \frac{[d d][d b]}{[a a]} \frac{\left(z-\frac{[b b]}{[d b]}\right)}{z}
$$

carries these branch points to

$$
\begin{array}{ll}
E_{1}=k \frac{[d a][d b]}{[a d]}, & \infty=\infty, \\
E_{2}=k \frac{[d c][d d]}{[a d]}, & E_{3}=k \frac{[d a][d c]}{[a a]}, \\
0=0, & E_{4}=k \frac{[d b][d d]}{[a a]},
\end{array}
$$

which is the expression (III.20). 
We convert Eq. (III.4) for $A, B, C, D$ to the notation of quadrics. Using (III.4) and (III.7), it follows that

$$
\begin{aligned}
\frac{2-A}{4} & =\frac{[d d][d a]}{[a a][a d]}, & \frac{2-B}{4} & =\frac{[d d][d b]}{[b b][b d]}, \\
\frac{2-C}{4} & =\frac{[d d][d c]}{[c c][c d]}, & D & =\frac{\alpha \beta \gamma \delta}{[d d]^{2}}(2-A)(2-B)(2-C) .
\end{aligned}
$$

Next, using (A.4), (A.7), (A.10) together with similar arguments to those above, we relate these Kummer parameters to branch points. These relations are expressed as cross ratios, so are invariant under linear fractional transformations,

$$
\begin{gathered}
\frac{2-A}{4}=\frac{\left(k_{1}-k_{5}\right)\left(k_{6}-k_{2}\right)}{\left(k_{1}-k_{2}\right)\left(k_{6}-k_{5}\right)}=\frac{\left(E_{1}-E_{3}\right)\left(E_{4}-E_{2}\right)}{\left(E_{1}-E_{2}\right)\left(E_{4}-E_{3}\right)}, \\
\frac{2-B}{4}=\frac{\left(k_{6}-k_{3}\right)\left(k_{4}-k_{5}\right)}{\left(k_{6}-k_{5}\right)\left(k_{4}-k_{3}\right)}=\frac{E_{4}}{E_{4}-E_{3}}, \\
\frac{2-C}{4}=\frac{\left(k_{3}-k_{2}\right)\left(k_{1}-k_{4}\right)}{\left(k_{3}-k_{4}\right)\left(k_{1}-k_{2}\right)}=\frac{-E_{2}}{E_{1}-E_{2}}, \\
D=4\left[\frac{\left(k_{3}-k_{6}\right)\left(k_{1}-k_{4}\right)\left(k_{2}-k_{4}\right)\left(k_{3}-k_{5}\right)-\left(k_{2}-k_{3}\right)\left(k_{4}-k_{5}\right)\left(k_{1}-k_{3}\right)\left(k_{4}-k_{6}\right)}{\left(k_{1}-k_{2}\right)\left(k_{3}-k_{4}\right)^{2}\left(k_{5}-k_{6}\right)}\right] \\
=\frac{4\left(E_{3} E_{4}-E_{1} E_{2}\right)}{\left(E_{1}-E_{2}\right)\left(E_{3}-E_{4}\right)} .
\end{gathered}
$$

\section{Appendix B. Kummer Varieties and the Jacobi Inversion Formulae}

This appendix has two purposes. First we establish Proposition II.4 concerning the number of connected components of $\mathscr{M}$. Our method uses the Kummer variety which is a higher dimensional analogue of the Kummer surface developed in Sect. III. In the second part we present a geometric derivation of the Jacobi inversion formulae (III.13a, b) for $N=2$. This derivation uses the orthogonality relations presented in III.C.

Both of these topics require some further discussion of the Abel-Jacobi map $\Psi$ (I.11). $\Psi$ is a holomorphic mapping from $\mathscr{R}^{(N)}=(\mathscr{R} \times \ldots \times \mathscr{R}) / \sigma_{N}$ onto $\mathscr{J}$. It is $1-1$ off of a codimension 1 subvariety of $\mathscr{R}^{(N)}$. This subvariety is contained in the inverse image of the theta divisor. Algebraically, this means that $\mathscr{J}$ is birational to $\mathscr{R}^{(N)}$.

For example, when $N=2, \Psi$ is $1-1$ off of the subvariety of hyperelliptic divisors, $\langle(\mu, y),(\mu,-y)\rangle$, in $\mathscr{R}^{(2)}$. This subvariety is isomorphic to $\mathbb{P}^{1}$, and, under $\Psi$, all of it maps to $\mathbf{0}$ in $\mathscr{J} ; \mathbf{0}$ is contained in the theta divisor of $\mathscr{J}$.

For general $N$ we can define a theta map

$$
\Phi: \mathscr{J} \rightarrow \mathbb{P}^{2^{N-1}}, \quad \mathbf{z} \mapsto\left[x_{1}(\mathbf{z}): \ldots: x_{2^{N}}(\mathbf{z})\right],
$$

where $x_{i}(\mathbf{z})=\Theta\left[\begin{array}{c}\varepsilon_{i} \\ 0\end{array}\right](2 \mathbf{z} ; 2 B)$. Here $\varepsilon_{i}=\left(\varepsilon_{i 1}, \ldots, \varepsilon_{i N}\right)$ with $\varepsilon_{i j}=0$ or 1 and $i$ indexes all $N$-tuples. As with the case $N=2$, this is a $2: 1$ map of $\mathscr{J}$ onto a $g$-dimensional algebraic variety called the Kummer variety, denoted by $S$. 
Since $\mathscr{J}$ is birational to $\mathscr{R}^{(N)}$, one can ask, what map on $\mathscr{R}^{(N)}$ corresponds to $\Phi$ ? From the definition of $\Psi$ one easily observes that identifying $\ell$ with $-\ell$ corresponds to identifying $\left\langle\left(\mu_{1}, y_{1}\right), \ldots,\left(\mu_{N}, y_{N}\right)\right\rangle$ with $\left\langle\left(\mu_{1},-y_{1}\right), \ldots,\left(\mu_{N},-y_{N}\right)\right\rangle$. Thus $\Phi$ corresponds to the quotient map

$$
\mathscr{R}^{(N)} \rightarrow \mathscr{R}^{(N)} /\{(\boldsymbol{\mu}, \mathbf{y}) \sim(\boldsymbol{\mu},-\mathbf{y})\} .
$$

One can go a step further with this and identify all branches:

$$
\left\langle\left(\mu_{1}, \pm y_{1}\right), \ldots,\left(\mu_{N}, \pm y_{N}\right)\right\rangle \text {. }
$$

In other words we map each point of $\mathscr{R}^{(N)}$ to its projected $\mu$-coordinates,

$$
\left(\mu_{1}, \ldots, \mu_{N}\right) \in \mathbb{P}^{1} \times \ldots \times \mathbb{P}^{1} / \sigma_{N} .
$$

$\mathbb{P}^{1} \times \ldots \times \mathbb{P}^{1} / \sigma_{N}$ is isomorphic to $\mathbb{P}^{N}$, complex projective $N$-space, since it is naturally coordinatized by the symmetric polynomials in the $\mu_{i}$ :

$$
\left[1: \sigma_{1}(\boldsymbol{\mu}): \sigma_{2}(\boldsymbol{\mu}): \ldots: \sigma_{N}(\boldsymbol{\mu})\right]=\left[1: \mu_{1}+\mu_{2}+\ldots+\mu_{N}: \ldots: \mu_{1} \mu_{2} \ldots \mu_{N}\right] .
$$

We summarize all of this in the following diagram:

Diagram 1

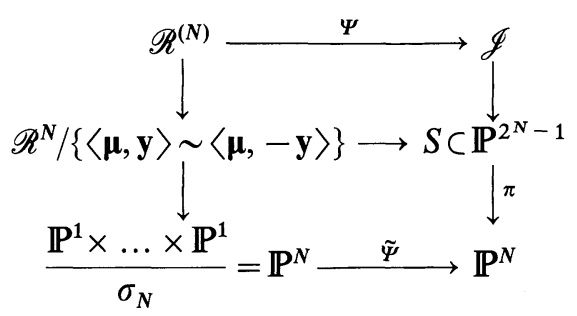

We now turn to the proof of Proposition II.4. A fundamental addition formula for quadratic theta functions is

$$
\Theta(\mathbf{z}+\mathbf{w}, B) \cdot \Theta(\mathbf{z}-\mathbf{w}, B)=\sum_{i=1}^{2^{N}} \Theta\left[\begin{array}{c}
\varepsilon_{i} \\
0
\end{array}\right](2 \mathbf{w}, 2 B) \Theta\left[\begin{array}{c}
\varepsilon_{i} \\
0
\end{array}\right](2 \mathbf{z}, 2 B) .
$$

Let $\alpha_{i}=\Theta\left[\begin{array}{c}\varepsilon_{i} \\ 0\end{array}\right](0,2 B)$ denote the theta nulls. Then from (B.3) we deduce

where $\left|\varepsilon_{i}\right|=\sum_{j=1}^{N} \varepsilon_{i j}$.

$$
\begin{aligned}
\Theta^{2}(\mathbf{z}) & =\sum_{i=1}^{2^{N}} \alpha_{i} x_{i}(\mathbf{z}), \\
\Theta^{2}\left(\mathbf{z}+\frac{\mathbf{1}}{2}\right) & =\sum_{i=1}^{2^{N}}(-1)^{\left|\varepsilon_{i}\right|} \alpha_{i} x_{i}(\mathbf{z}),
\end{aligned}
$$

From the theta quotient representation of $u$, we then have

$$
e^{-i u}=\frac{\Theta^{2}\left(\ell+\Delta+\frac{1}{2}\right)}{\Theta^{2}(\ell+\Delta)}=\frac{\sum_{i=1}^{2^{N}}(-1)^{\left|\varepsilon_{i}\right|} \alpha_{i} x_{i}}{\sum_{i=1}^{2^{N}} \alpha_{i} x_{i}} .
$$

For $u$ real, this quotient must have modulus 1 . However, this quotient has the form

$$
\frac{\Sigma_{1}-\Sigma_{2}}{\Sigma_{1}+\Sigma_{2}},
$$


where $\Sigma_{1}=\sum_{\left|\varepsilon_{i}\right| \equiv 0(\bmod 2)} \alpha_{i} x_{i}$ and $\Sigma_{2}=\sum_{\left|\varepsilon_{i}\right| \equiv 1(\bmod 2)} \alpha_{i} x_{i}$. In order for a quotient of this form to have modulus 1 it is necessary that

$$
\Sigma_{1}=r_{1} e^{i \theta}, \quad \Sigma_{2}=i r_{2} e^{i \theta},
$$

where $r_{j}, \theta$ are real. Since the $x_{i}$ are coordinates in projective space we can uniformly divide them by $e^{i \theta}$. Then

and

$$
e^{-i u}=\frac{r_{1}-i r_{2}}{r_{1}+i r_{2}}
$$

$$
\left(\Theta^{2}\left(\ell+\Delta+\frac{1}{2}\right)\right)^{*}=\Theta^{2}(\ell+\Delta)
$$

if $\mathbf{u}$ is real. This suffices to establish Proposition II.4.

Next we will derive the Jacobi inversion formulae for $N=2$. Wee seek a rational map $\mathbb{P}^{3} \rightarrow \mathbb{P}^{2}$ which induces the map $\pi: S \rightarrow \mathbb{P}^{2}$ indicated in Diagram 1 . This done, the reader will see that we have reduced the inversion of the non-linear map $\Psi$ to the, comparatively, trivial inversion of a linear map $\tilde{\Psi}$.

The map $\pi: S \rightarrow \mathbb{P}^{2}$ in Diagram 1 represents $S$ as a hyperelliptic surface; i.e., $S$ is a 2:1 covering of $\mathbb{P}^{2}$ branched along a curve. This branch locus uniquely determines the map $S \rightarrow \mathbb{P}^{2}$. We will determine the map by finding the branch locus of the corresponding map on the left-hand side of Diagram 1; this map fails to be $2: 1$ (i.e., it branches) along the union of curves

$$
\begin{array}{lll}
\mathbb{P}^{1} \times\{0\}, & \mathbb{P}^{1} \times\{\infty\}, & \mathbb{P}^{1} \times\left\{E_{1}\right\}, \\
\mathbb{P}^{1} \times\left\{E_{2}\right\}, & \mathbb{P}^{1} \times\left\{E_{4}\right\}, & \mathbb{P}^{1} \times\left\{E_{3}\right\},
\end{array}
$$

in $\mathscr{R}^{(2)} /\{\sim\}$. Under $\Phi \circ \Psi$ these curves map to tropes as follows:

$$
\left.\begin{array}{l}
\mathbb{P}^{1} \times\{0\} \rightarrow(a c) \equiv\{-\delta x-\gamma y+\beta z+\alpha w=0\}, \\
\mathbb{P}^{1} \times\{\infty\} \rightarrow(a b) \equiv\{-\delta x+\gamma y-\beta z+\alpha w=0\}, \\
\mathbb{P}^{1} \times\left\{E_{1}\right\} \rightarrow(b c) \equiv\{-\gamma x-\delta y+\alpha z+\beta w=0\}, \\
\mathbb{P}^{1} \times\left\{E_{2}\right\} \rightarrow(b a) \equiv\{\gamma x-\delta y-\alpha z+\beta w=0\}, \\
\mathbb{P}^{1} \times\left\{E_{3}\right\} \rightarrow(c a) \equiv\{\beta x-\alpha y-\delta z+\gamma w=0\}, \\
\mathbb{P}^{1} \times\left\{E_{4}\right\} \rightarrow(c b) \equiv\{-\beta x+\alpha y-\delta z+\gamma w=0\} .
\end{array}\right\}
$$

One observes that these are precisely the six tropes containing the node $[\alpha: \beta: \gamma: \delta]=\Phi(\mathbf{0})$. A $2: 1$ map $S \rightarrow \mathbb{P}^{2}$ branched along these six tropes can be constructed by stereographic projection from the node $[\alpha: \beta: \gamma: \delta]$. To do this we choose a basis for the linear space of all planes containing $[\alpha: \beta: \gamma: \delta]$. This is a 3 dimensional space. Projectively, it is $\mathbb{P}^{2}$. As a basis for this space we choose 3 tropes: $x_{0}=(a c), x_{1}=(b a), x_{2}=(c b)$. Using this basis, the stereographic projection is defined by

$$
\mathbb{P}^{3} \rightarrow \mathbb{P}^{2}
$$

$$
\begin{aligned}
& {[x: y: z: w] \mapsto\left[x_{0}: x_{1}: x_{2}\right]} \\
& \quad=[-\delta x-\gamma y+\beta z+\alpha w: \gamma x-\delta y-\alpha z+\beta w:-\beta x+\alpha y-\delta z+\gamma w] .
\end{aligned}
$$


Since $S$ is a surface of degree 4, and we are projecting from a node (which is a point of multiplicity 2), the map (B.6) restricted to $S$ is a $2: 1$ map onto $\mathbb{P}^{2}$. A priori, the value of this map at $[\alpha: \beta: \gamma: \delta]$ is not defined because $x_{0}, x_{1}$, and $x_{2}$ all vanish there. We must blow-up $S$ at this node so that the map (B.6) extends to be defined on the projectivized tangent cone at this node. As a result of blowing-up we recover the exceptional locus that was blown down by the Abel-Jacobi map. Thus, the map $\mathbb{P}^{2} \stackrel{\tilde{\Psi}}{\longrightarrow} \mathbb{P}^{2}$ will in fact be biholomorphic, not just birational. However, all biholomorphic maps $\mathbb{P}^{n} \rightarrow \mathbb{P}^{n}$ are in fact linear maps [23]. Hence, to determine $\tilde{\Psi}$ it will suffice to know its values on a basis for $\mathbb{P}^{2}$.

In the symmetric $\boldsymbol{\mu}$-coordinates (B.1), which we denote $\left[z_{0}: z_{1}: z_{2}\right]$ $=\left[1: \mu_{1}+\mu_{2}: \mu_{1} \mu_{2}\right]$, we represent the branch curves:

$$
\begin{aligned}
& \mathbb{P}^{1} \times\{0\}=\left\{[1: \mu: 0] \mid \mu \in \mathbb{P}^{1}\right\} \equiv\left\{z_{2}=0\right\}, \\
& \mathbb{P}^{1} \times\{\infty\}=\left\{\left[0: \frac{1}{\mu}: 1\right] \mid \mu \in \mathbb{P}^{1}\right\} \equiv\left\{z_{0}=0\right\}, \\
& \mathbb{P}^{1} \times\left\{E_{1}\right\}=\left\{\left[1: E_{1}+\mu: \mu E_{1}\right] \mid \mu \in \mathbb{P}^{1}\right\} \equiv\left\{E_{1} z_{0}-z_{1}+\frac{1}{E_{1}} z_{2}=0\right\}, \\
& \mathbb{P}^{1} \times\left\{E_{2}\right\}=\left\{\left[1: E_{2}+\mu: \mu E_{2}\right] \mid \mu \in \mathbb{P}^{1}\right\} \equiv\left\{E_{2} z_{0}-z_{1}+\frac{1}{E_{2}} z_{2}=0\right\}, \\
& \mathbb{P}^{1} \times\left\{E_{3}\right\}=\left\{\left[1: E_{3}+\mu: \mu E_{3}\right] \mid \mu \in \mathbb{P}^{1}\right\} \equiv\left\{E_{3} z_{0}-z_{1}+\frac{1}{E_{3}} z_{2}=0\right\}, \\
& \mathbb{P}^{1} \times\left\{E_{4}\right\}=\left\{\left[1: E_{4}+\mu: \mu E_{4}\right] \mid \mu \in \mathbb{P}^{1}\right\} \equiv\left\{E_{4} z_{0}-z_{1}+\frac{1}{E_{4}} z_{2}=0\right\} .
\end{aligned}
$$

$\widetilde{\Psi}$ maps these six lines to the stereographic projections of the corresponding tropes (B.5). Since these tropes are conics (curves of degree 2) containing $[\alpha: \beta: \gamma: \delta]$, projection from this point maps these tropes to degree 1 curves, i.e. to lines in $\mathbb{P}^{2}$. Since they are lines, their defining forms are linear combinations of the coordinates $x_{0}, x_{1}, x_{2}$ (B.6). The coefficients in these linear equations turn out to be fundamental quadrics (III.7). We present here the equations of these projected tropes in $\left[x_{0}: x_{1}: x_{2}\right]$-coordinates. The reader may check the correctness of these equations by direct inspection,

$$
\begin{aligned}
& \mathbb{P}^{1} \times\{0\} \rightarrow\left\{x_{0}=0\right\}, \\
& \mathbb{P}^{1} \times\{\infty\} \rightarrow\left\{[d a] x_{0}-[c c] x_{1}+[b d] x_{2}=0\right\}, \\
& \mathbb{P}^{1} \times\left\{E_{1}\right\} \rightarrow\left\{[c d] x_{0}+[b d] x_{1}-[a a] x_{2}=0\right\}, \\
& \mathbb{P}^{1} \times\left\{E_{2}\right\} \rightarrow\left\{x_{1}=0\right\}, \\
& \mathbb{P}^{1} \times\left\{E_{3}\right\} \rightarrow\left\{-[b b] x_{0}+[a d] x_{1}+[d c] x_{2}=0\right\}, \\
& \mathbb{P}^{1} \times\left\{E_{4}\right\} \rightarrow\left\{x_{2}=0\right\} .
\end{aligned}
$$


Since $\tilde{\Psi}$ must map each branch curve in $\left[z_{0}: z_{1}: z_{2}\right]$-space to its correspondent in $\left[x_{0}: x_{1}: x_{2}\right]$-space, we see from comparison of (B.7) with (B.8) that

$$
\left.\begin{array}{l}
x_{0}=\lambda_{0} \tilde{\Psi}\left(z_{2}\right), \\
x_{1}=\lambda_{1} \tilde{\Psi}\left(E_{2} z_{0}-z_{1}+\frac{1}{E_{2}} z_{2}\right), \\
x_{2}=\lambda_{2} \tilde{\Psi}\left(E_{4} z_{0}-z_{1}+\frac{1}{E_{4}} z_{2}\right),
\end{array}\right\}
$$

$\lambda_{0}, \lambda_{1}, \lambda_{2}$ are unspecified scalars. Of course, we have only used three branch curves here. By substituting the expressions for $x_{i}$ given by (B.9) into the remaining linear equations of (B.8) and then comparing these with (B.7), we can deduce the $\lambda_{i}$-values.

To do this we replace the $E_{i}$ 's in (B.7) and (B.9) by their expressions in terms of fundamental quadrics as given by (III.20) [or (A.9)]. Thus (B.9) becomes

$$
\begin{aligned}
& x_{0}=\lambda_{0} z_{2}, \\
& x_{1}=\lambda_{1}\left(k \frac{[d c][d d]}{[a d]}\right) z_{0}-\lambda_{1} z_{1}+\lambda_{1}\left(\frac{1}{k} \frac{[a d]}{[d c][d d]}\right) z_{2}, \\
& x_{2}=\lambda_{2}\left(k \frac{[d b][d d]}{[a a]}\right) z_{0}-\lambda_{2} z_{1}+\lambda_{2}\left(\frac{1}{k} \frac{[a a]}{[d b][d d]}\right) z_{2} .
\end{aligned}
$$

(We have abused notation and dropped $\tilde{\Psi}$ here. This causes no problem because $\tilde{\Psi}$ is linear.) Comparing the second linear equation in (B.7) and (B.8) $\left(\mathbb{P}^{1} \times \infty\right)$ we have

$$
[d a] x_{0}-[c c] x_{1}+[b d] x_{2}=z_{0} .
$$

Substituting (B.10) into (B.11) we derive

$$
\begin{array}{r}
\lambda_{0}[d a]-\frac{\lambda_{1}}{k} \frac{[c c][a d]}{[d c][d d]}+\frac{\lambda_{2}}{k} \frac{[b d][a a]}{[d b][d d]}=0, \\
\lambda_{1}[c c]-\lambda_{2}[b d]=0 .
\end{array}
$$

Since, $\tilde{\Psi}$ is a projective linear map, we may take $\lambda_{1}=1$. It then follows from (B.12) that

$$
\begin{aligned}
& \lambda_{0}=\frac{1}{k} \frac{[b b][c c][c d]}{[d a][d b][d c][d d]}, \\
& \lambda_{1}=1, \\
& \lambda_{2}=\frac{[c c]}{[b d]} .
\end{aligned}
$$


Substituting (B.13) into (B.10) we can finally write down $\tilde{\Psi}$ :

$$
\tilde{\Psi}=\left[\begin{array}{ccc}
0 & 0 & \frac{1}{k} \frac{[b b][c c][c d]}{[d a][d b][d c][d d]} \\
k \frac{[d c][d d]}{[a d]} & -1 & \frac{1}{k} \frac{[a d]}{[d c][d d]} \\
k \frac{[c c][d b][d d]}{[b d][a a]} & -\frac{[c c]}{[b d]} & \frac{1}{k} \frac{[c c][a a]}{[b d][d b][d d]}
\end{array}\right]
$$

Our goal is now before us. We have reduced the Jacobi inversion problem to the inversion of the matrix $\tilde{\Psi}$. This is straightforward. The tedious manipulation of symbolic expessions which arise in this inversion is greatly facilitated by repeated use of the orthogonality relations among the fundamental quadrics (III.C). The result is:

$$
\tilde{\Psi}^{-1}=\left[\begin{array}{ccc}
\frac{1}{k} \frac{[d a][a a][a d]}{[b b][c c][c d][d d]} & -\frac{1}{k} \frac{[a a][a d]}{[b b][c d][d d]} & \frac{1}{k} \frac{[a a][b d][a d]}{[b b][c c][c d][d d]} \\
\frac{[d a]\{[a a][d c]+[a d][d b]\}}{[b b][c c][c d]} & -\frac{[d b][a d]}{[b b][c d]} & \frac{[d c][a a][b d]}{[b b][c c][c d]} \\
k \frac{[d a][d b][d c][d d]}{[b b][c c][c d]} & 0 & 0
\end{array}\right] .
$$

Since $\left[z_{0}: z_{1}: z_{2}\right]^{T}=\tilde{\Psi}^{-1} \circ\left[x_{0}: x_{1}: x_{2}\right]^{T}$ and $\left[x_{0}: x_{1}: x_{2}\right]=[(a c):(b a):(c b)]$, we deduce

$$
\left.\begin{array}{rl}
z_{0}= & \frac{1}{k} \frac{[a a][a d]}{[b b][c c][c d][d d]}\{[d a](a c)-[c c](b a)+[b d](c b)\} \\
= & \frac{1}{k} \frac{[a a][a d]}{[b b][c c][c d]}(a b), \\
z_{1}= & \frac{1}{[b b][c c][c d]}\{[d a]\{[a a][d c]+[a d][d b]\} \\
& \cdot(a c)-[a d][d b][c c](b a)+[a a][b d][d c](c b)\}, \\
z_{2}= & k \frac{[d a][d b][d c][d d]}{[b b][c c][c d]}(a c) .
\end{array}\right\}
$$

From (B.1), we observe that $\mu_{1}+\mu_{2}=z_{1} / z_{0}$ and $\mu_{1} \mu_{2}=z_{2} / z_{0}$. Hence,

$$
\begin{aligned}
\mu_{1}+\mu_{2} & =\frac{[d a]\left\{\frac{[d c]}{[a d]}+\frac{[d b]}{[a a]}\right\}(a c)-\frac{[d b][c c]}{[a a]}(b a)+\frac{[b d][d c]}{[a d]}(c b)}{(a b)} \\
\mu_{1} \mu_{2} & =k^{2} \frac{[d a][d b][d c][d d]}{[a a][a d]} \frac{(a c)}{(a b)}
\end{aligned}
$$


Using (III.10) and the orthogonality relations, we deduce the

\section{Inversion Formulae (III.13).}

$$
\begin{aligned}
\mu_{1}+\mu_{2} & =\frac{[d a]\left\{E_{2}+E_{4}\right\}(a c)-[c c] E_{4}(b a)+[b d] E_{2}(c b)}{[d d](a b)}, \\
\mu_{1} \mu_{2} & =\sqrt{E_{1} E_{2} E_{3} E_{4}} \frac{(a c)}{(a b)} .
\end{aligned}
$$

Acknowledgements. Both authors have benefitted significantly from discussions with Dave McLaughlin which helped to motivate and clarify ideas. We thank Joe Neisendorfer for discussions relating to topology. We also thank the referee for his thorough and helpful review.

\section{References}

1. Lamb, G.L., Jr.: Analytical descriptions of ultrashort optical pulse propagation in a resonant medium. Rev. Mod. Phys. 43, 99

2. (a) Ablowitz, M.J., Kaup, D.: Method for solving the sine-Gordon equation. Phys. Rev. Lett. 30, 1262

(b) Ablowitz, M.J., Kaup, D., Newell, A.: Coherent pulse propagation, a dispersive, irreversible phenomenon. J. Math. Phys. 15, 1852 (1974)

3. Faddeev, L.D., Takhtajian, L.A.: Essentially nonlinear one-dimensional model of classical field theory. Theor. Math. Phys. 21, 1046 (1974)

4. Forest, M.G., McLaughlin, D.W.: Spectral theory, for the periodic sine-Gordon equation: a concrete viewpoint. J. Math. Phys. 23 (7), 1248-1277 (1982)

5. McKean, H.P.: The sine-Gordon and sinh-Gordon equations on the circle. C.P.A.M. 34, 197-257 (1981)

6. Forest, M.G., McLaughlin, D.W.: Modulations of sine-Gordon and sinh-Gordon wavetrains. Stud. Appl. Math. 68, 11-59 (1983)

7. Dubrovin, B.A., Matveev, V.B., Novikov, S.P.: Nonlinear equations of Korteweg-de Vries type, finite-zone linear operators, and abelian varieties. Usp. Mat. Nauk. 31, 55-136 (1976)

8. Matveev, V.B.: Abelian functions and solitons. Preprint No. 373, University of Wroclaw (1976)

9. Daté, E.: Multisoliton solutions and quasi-periodic solutions of nonlinear equations of sineGordon type. Preprint, 1981

10. Daté, E., Tanaka, S.: Periodic multi-soliton solutions of the Korteweg-de Vries equation and Toda lattice. Prog. Theor. Phys. Suppl. 59, 107-125 (1976)

11. Forest, M.G., McLaughlin, D.W.: Canonical variables for the periodic sine-Gordon equation and a method of averaging. LA-UR 78-3318, Report of Los Alamos Scientific Laboratory, 1978

12. Siegel, C.L.: Topics in complex function theory, Vol. II. New York: Wiley-Interscience 1971

13. Dubrovin, B.A., Natanson, S.M.: Real two-zone solutions of the sine-Gordon equation. Funk. Anal. i Ego Pril 16 (1), 27-43 (1982)

14. McKean, H.P., van Moerbeke, P.: The spectrum of Hill's equation. Invent. Math. 30, 217-274 (1975)

15. Birnir, B.: Ph.D. Thesis, N.Y.U., 1980

16. Enol'skii, V.Z., Belokolos, E.D.: Classification of nonlinear waves in the Josephson junction. Preprint of Inst. Theor. Phys., Acad. Sci. Ukrain., 1981, and subsequent preprint, ITP-82-36E, 1982

17. Ercolani, N.M., Forest, M.G., McLaughlin, D.W.: Modulational stability of two-phase sineGordon wavetrains. Stud. Appl. Math. 71, 91-101 (1984) 
18. Ercolani, N.M., Forest, M.G., McLaughlin, D.W.: Modulational instabilities of periodic sine Gordon waves: a geometric analysis. To appear in nonlinear systems of PDE in Appl. Math., AMS-SIAM Summer Seminar, 1984

19. Picard, E.: Theorie des courbes et des surfaces algébriques. Paris: Gauthier-Villars 1931

20. (a) Kaup, D.J.: J. Math. Anal. Appl. 54, 849 (1976)

(b) McLaughlin, D.W., Scott, A.C.: Perturbation analysis of fluxon dynamics. Phys. Rev. A 18, 1652-1680 (1978)

21. Rauch, H., Farkas, H.: Theta functions with applications to Riemann surfaces. Baltimore: Williams and Wilkins 1974

22. Hudson, W.H.T.: Kummer's quartic surface. Cambridge: Cambridge University Press 1905

23. Griffiths, P., Harris, J.: Principles of algebraic geometry. New York: Wiley 1978

24. Whitham, G.B.: Linear and nonlinear waves. New York: Wiley 1974

25. Sommerfeld, A.: Optics. New York: Academic Press 1964

26. Lamb, G.L., Jr.: Elements of soliton theory. New York: Wiley 1980

27. Flaschka, H., Forest, M.G., McLaughlin, D.W.: Multiphase averaging and the inverse spectral solution of the KdV equation. Comm. Pure Appl. Math. 33, 739-784 (1980)

28. Previato, E.: Harvard Doctoral Thesis, 1983

29. Deift, P., Lund, F., Trubowitz, E.: Nonlinear wave equations and constrained harmonic motion. Commun. Math. Phys. 74, 141-188 (1980)

Communicated by S.-T. Yau

Received January 10, 1983; in revised form November 9, 1984 
